\title{
Sobre la generalización de algunos teoremas de geometría
}

\author{
M.A. Murray-Lasso \\ Unidad de Enseñanza Auxiliada por Computadora \\ Departamento de Ingeniería de Sistemas. División de Estudios de Posgrado \\ Facultad de Ingeniería, UNAM
}

(recibido: abril de 2001; aceptado: julio de 2001)

\begin{abstract}
Resumen
Utilizando tres tipos de herramientas (herramientas convencionales de geometría elemental, tales como triángulos semejantes; números complejos y vectores tridimensionales), se analizan cuidadosamente las demostraciones de dos famosos teoremas de Geometría: el Teorema de Pitágoras y el Teorema de Varignon, y se toma nota de cuál es el alcance de la validez de la demostración para establecer que los dos famosos teoremas tengan un rango de validez mayor del que normalmente se les asigna. De esta manera, se establecen teoremas extendidos de Pitágoras y Varignon. Los resultados principales son que no solamente las áreas de los cuadrados construidos sobre los catetos de un un triángulo rectángulo se suman para obtener el área del cuadrado construido sobre la hipotenusa, sino que el teorema es válido para las áreas de cualesquiera figuras semejantes que se construyan sobre los lados del triángulo rectángulo: círculos, pentágonos regulares, flores de lis, efigies del águila devorando una serpiente, y en general, cualquier figura plana para la cual esté bien definido el concepto de área. En cuanto al Teorema de Varignon, se establecen dos familias de teoremas sobre figuras semejantes muy generales, que incluyen diversos teoremas asociados con construcciones de figuras específicas sobre los lados de un cuadrilátero para encontrar vértices de paralelogramos. Se eliminan condiciones de convexidad. Dichos teoremas son también válidos para triángulos considerados como cuadriláteros con un lado con longitud cero. Se generaliza y extiende el Teorema de Varignon a cuadriláteros en el espacio tridimensional, introduciendo transformaciones lineales generales, de estas las afines, rotaciones, reflexiones y traslaciones son casos particulares. Se ilustra además cómo con herramientas más abstractas se logra la generalización de manera similar a las generalizaciones que se han hecho de los números (naturales, enteros positivos y negativos, racionales, irracionales, reales y complejos).
\end{abstract}

Descriptores: Pitágoras, Varignon, números complejos, vectores, transformación y cuadrilátero.

\section{Abstract}

Using three types of tools (conventional tools of elementary geometry, such as similar triangles; complex numbers and three-dimensional vectors), the proofs of two famous theorems in geometry, namely the Therem of Pythagoras and the Theorem of Varignon, are carefully analyzed and note is taken of what is the range of validity of the proof in order to establish that the two famous theorems have a range of validity larger than normally assigned to them. The principal results are that not only the areas of squares constructed on the legs of a right angled triangle can be added to give the area of the square constructed on the hypothenuse, but the theorem is valid for the areas of any similar figures so constructed whether they be circles, regular pentagons, fleurs de lys, effigies of an eagle eating a serpent, and in general any flat figure for which the concept of area is well defined. As regards the Theorem of Varignon, two families of theorems are established based on a very general class of similar figures which encompasses several theorems connected to the construction of specific figures on the sides of a quadrilaterals in order to find the vertices of a parallelogram. Convexity conditions are eliminated. The theorems mentioned are also applicable to triangles considered as special cases of quadrilaterals with one side of length zero. The theorem of Varignon is generalized and extended to quadrilaterals in three 


\begin{abstract}
dimensional space introducing general linear transformations of which affine, rotations, reflections and traslations are specific cases.

It is illustrated how, using more abstract tools, a generalization, akin to that of natural numbers to positive and negative integers, rational numbers, real numbers and complex numbers, is acomplished for some areas of geometry.
\end{abstract}

Keywords: Pythagoras, Varignon, complex numbers, vectors, transformations, cuadrilateral.

\section{Introducción}

En este artículo se intentará resaltar la importancia de dos ideas:

1. Vale la pena tener más de un método para resolver un problema. Todos los métodos son dignos de estudio, pues pueden aportar enfoques complementarios.

2. Donde hay una buena idea generalmente hay más buenas ideas cercanas. Las demostraciones bien analizadas con diversas herramientas pueden llevar a extensiones y generalizaciones que expliquen mejor un área del conocimiento.

Para establecer la importancia de estas ideas se darán algunos ejemplos de su aplicación en el área de la geometría, tanto plana como tridimensional. Uno de los resultados es una nueva familia de teoremas sobre cuadriláteros en el plano y en el espacio.

En este artículo vamos a exhibir algunas demostraciones de teoremas conocidos y se comentará sobre la complejidad de los mismos, así como qué tanto se prestan a sugerir e inspirar extensiones y generalizaciones. Esta cuestión depende, naturalmente, de la persona sugerida o inspirada; a algunos una demostración les puede inspirar una generalización y a otros quizás no. Asimismo, una demostración debe ir en el contexto de una secuencia de teoremas previamente demostrados en los cuales se apoya. Dada la imposibilidad, por falta de espacio, de exhibir las secuencias completas, se hacen frecuentes referencias a libros de texto estándar, los cuales pueden ser consultados por los lectores para ver en qué axiomas y teoremas previos se basa una demostración, ya que ni siquiera los axiomas coinciden totalmente en todos los tratados, aunque en términos generales siguen los Elementos de Euclides.

\section{El Teorema de Pitágoras y su extensión}

El Teorema de Pitágoras (580? - 500? A. C.) es uno de los teoremas más importantes de la Geometría. El Prof. Wylie C.R. Jr. (1964), dice en su libro Foundations of Geometry, refiriéndose al Teorema de Pitágoras: “... el teorema probablemente más famoso y más importante de las matemáticas". Davis y Hersh (1981) dicen: "... el que es sin duda el más famoso teorema en la historia de las matemáticas tal como aparece en el más famoso libro en la historia de las matemáticas ..." al referirse al teorema de Pitágoras que aparece en la Proposición 47 del Libro I de los Elementos de Euclides (300 A.C.). El teorema como se enseña hoy en día en la educación media dice: "En cualquier triángulo rectángulo el cuadrado de la hipotenusa es igual a la suma de los cuadrados de los catetos". (Jurgensen, et al., 1963) y (Moise y Downs, 1975). Para este teorema, al pasar de los años se han dado cientos de demostraciones (Jurgensen, et al., 1963). La manera como lo conocían los antiguos griegos era: "El área de un cuadrado sobre la hipotenusa de un triángulo rectángulo es igual a la suma de las áreas de los cuadrados sobre sus catetos". (Moise y Downs, 1975). La figura 1 ilustra esta situación. Los de esa época no conocieron el álgebra, por lo que el término "cuadrado" es geométrico, no algebraico o funcional. Fue el álgebra la que heredó siglos después la palabra "cuadrado" para referirse a la segunda potencia.

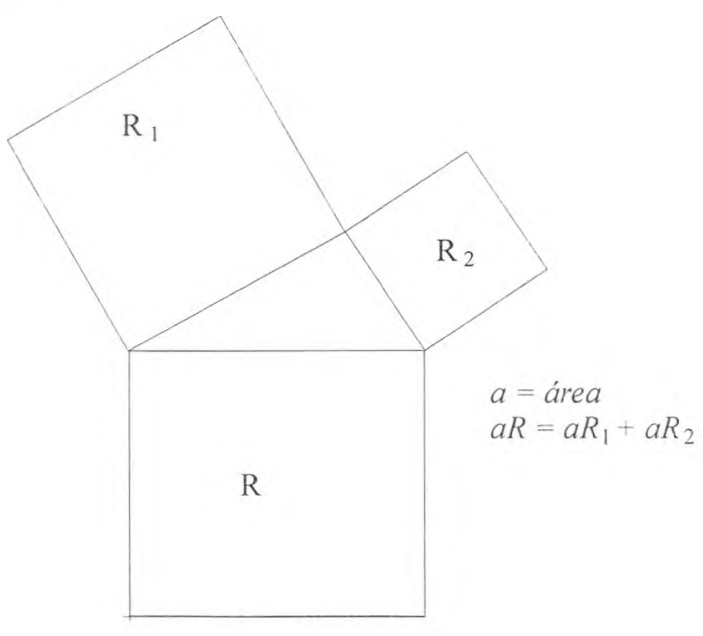

Figura 1

De las centenas de demostraciones existentes del teorema de Pitágoras mostraremos tres para ilustrar cómo una demostración puede inspirar una generalización más que otra. Comenzamos por la demostración que aparece en los Elementos de Euclides, escrita alrededor del año 300 A.C. Considérese la figura 2. 


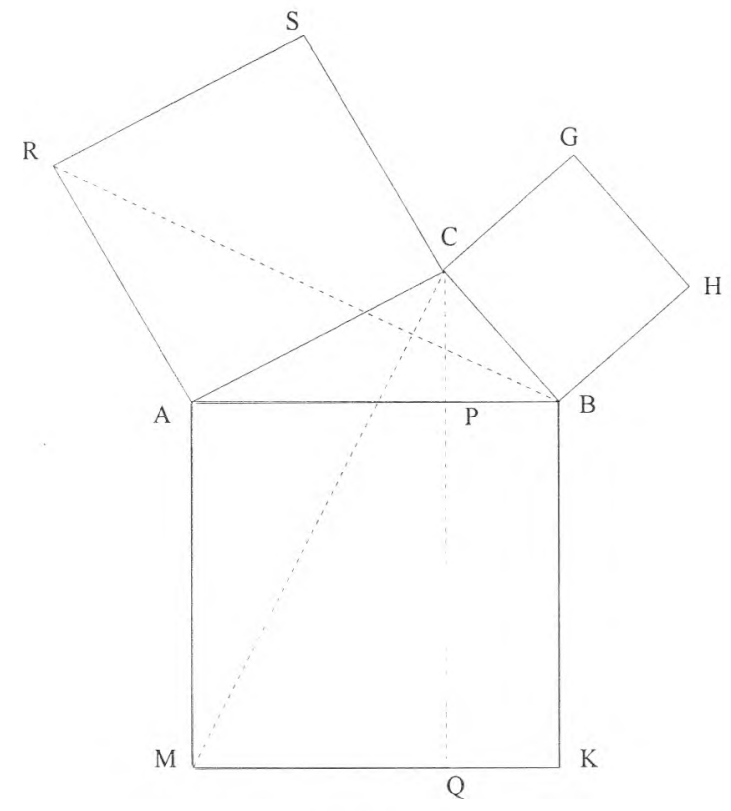

Figura 2

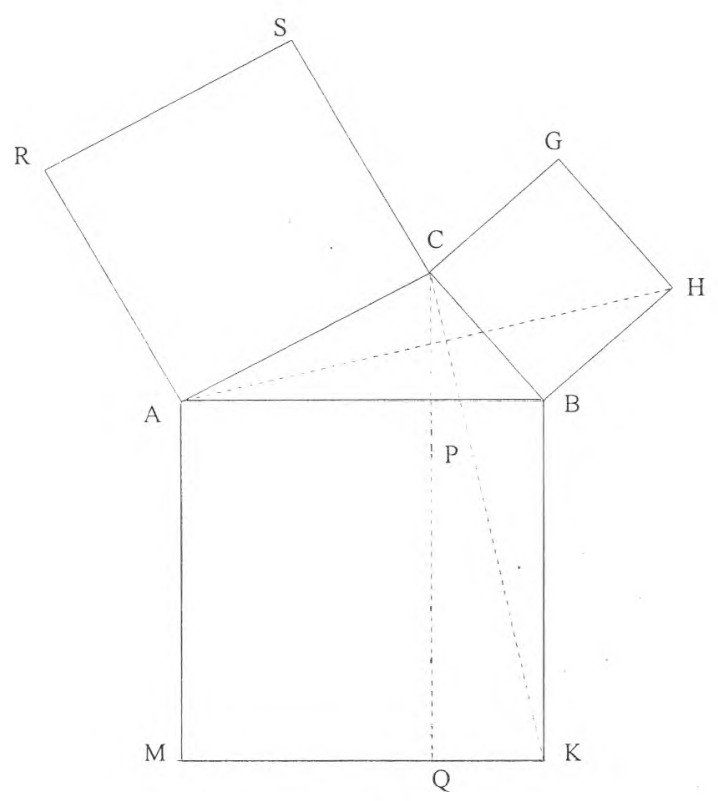

Figura 3

Por construcción los segmentos CQ y AM son paralelos; AS, $\mathrm{CH}$ y $\mathrm{MB}$ son cuadrados, cuyos lados tienen longitudes que son iguales a las longitudes de los lados $\mathrm{AC}, \mathrm{CB}$ y AB del triángulo $\mathrm{ACB}$ que es rectángulo con el ángulo recto en $\mathrm{C}$.

Los triángulos MAC y BAR tienen sus ángulos obtusos en $A$ iguales, pues ambos valen un ángulo recto más el ángulo BAC. Los lados $\mathrm{AB}$ y $\mathrm{AM}$ son congruentes, pues son lados de un cuadrado. Los lados AC y AR son congruentes por la misma razón. Por lo tanto, los triángulos BAR y MAC son congruentes por el teorema lado - ángulo - lado y por lo tanto tienen la misma área. El área del cuadrado AS es igual al doble del área del triángulo BAR, dado que el segmento AR es base para ambos y tienen como altura la longitud del segmento AC. El área del rectángulo AQ es el doble del área del triángulo MAC, ya que el segmento AM es la base de ambos y el segmento AP la altura de ambos. Podemos entonces concluir que las áreas del cuadrado AS y del rectángulo $\mathrm{AQ}$ son iguales, ya que ambas son el doble de las áreas de triángulos congruentes.

Si ahora trazamos las rectas punteadas $\mathrm{AH}$ y $\mathrm{CK}$, como se muestra en la figura 3, y aplicamos el mismo argumento a los triángulos $\mathrm{ABH}$ y $\mathrm{KBC}$ encontraremos que son congruentes y tienen la misma área, y que las áreas del cuadrado $\mathrm{CH}$ y del rectángulo $\mathrm{BQ}$ son ambas iguales al doble del área común de los triángulos mencionados, razón por la cual las áreas del cuadrado $\mathrm{CH}$ y del rectángulo $\mathrm{BQ}$ son iguales.

Finalmente, en vista de que el área del cuadrado AK sobre la hipotenusa es igual a la suma de las áreas de los rectángulos $\mathrm{AQ}$ y $\mathrm{BQ}$, las cuales a su vez son iguales a las áreas de los cuadrados AS y CH sobre los catetos, finalmente llegamos al resultado: El área del cuadrado sobre la hipotenusa del triángulo rectángulo es igual a la suma de las áreas de los cuadrados sobre sus catetos. Esto es lo que se deseaba demostrar.

Vemos que aunque un poco complicada la demostración se centra en demostrar que la suma de las áreas de ciertas figuras construidas (cuadrados) sobre los catetos es igual al área de una figura similar (otro cuadrado) construido sobre la hipotenusa. Seguramente, antes de intentar la demostración los antiguos griegos ya sabían el resultado al que querían llegar, por lo que de una buena vez le agregaron a la figura sobre la que se iba a razonar, los cuadrados cuyas áreas se iban a sumar. Adicionalmente, agregaron varias líneas auxiliares que no tienen que ver con el enunciado del teorema, pero que son útiles para establecer los puentes que relacionan los conceptos que sí entran en el enunciado.

Reproducimos ahora una segunda demostración del Teorema de Pitágoras, más elegante que la anterior, pero que difícilmente inspira la extensión que vamos a hacer posteriormente en este artículo. Se dice que esta demostración se debe a un humilde hindú que vivió hace siglos (Jurgensen et al., 1975).

En la figura 4 se muestra un cuadrado con cuatro triángulos inscritos con las características que se describen, con cateto menor de longitud $a$, cateto mayor de longitud $b$, e hipotenusa de longitud $c$, que fue colocado como se indica en la figura, en cuatro posiciones tales que, tanto el marco exterior como el interior son cuadrados. El exterior tiene lado de longitud $c$ y el del interior tiene lado de longitud $b-$ a. El marco exterior es cuadrado y se demuestra observando que los ángulos agudos de un triángulo rectángulo son complementarios, por lo que en las cuatro esquinas se tienen ángulos rectos. Que el cuadrilátero interno es un cuadrado 
es consecuencia de que los cuatro triángulos tienen su ángulo recto como ángulos exteriores del cuadrilátero, apoyados en la misma recta de uno de los catetos, y que los cuatro lados del cuadrilátero interior tienen la misma longitud $b-a$.

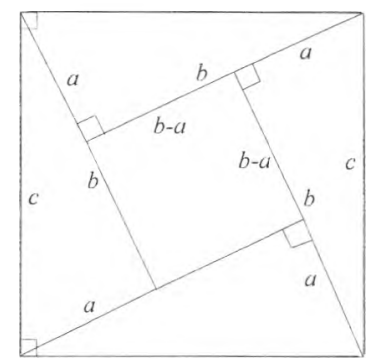

\section{Figura 4}

Haciendo un balance de áreas de la figura se tiene:

$$
\begin{gathered}
c^{2}=4(a b / 2)+(b-a)^{2}=2 a b+b^{2}+a^{2}-2 a b \\
=b^{2}+a^{2}
\end{gathered}
$$

El primer y último miembro dan

$$
c^{2}=a^{2}+b^{2}
$$

que es la expresión que se quería demostrar.

La demostración es considerablemente más corta y elegante que la anterior, no hubo necesidad de trazar líneas auxiliares, aunque si se requirió ingenio para concebir la figura original; sin embargo, la demostración se presta poco a la extensión que tenemos en mente pues, como la demostración de Euclides, está de antemano orientada a que las figuras que se construyen sobre los lados del triángulo son cuadrados.

La tercera demostración que exhibimos es todavía más elegante que la anterior y tiene la ventaja que no está orientada a cuadrados, razón por la cual inspira presentar el teorema en forma más general. Dicha demostración aparece en Coxeter (1971), donde se cita a Heath (1956).

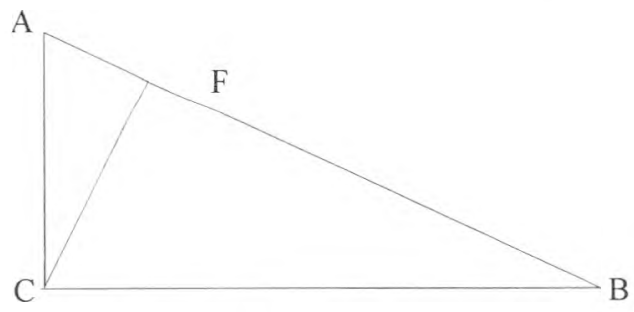

Figura 5

En el triángulo rectángulo $\mathrm{ABC}$ que se muestra en la figura 5, trácese desde el ángulo recto una perpendicular a la hipotenusa. Se tienen tres triángulos rectángulos semejantes: (escribiendo los vértices homólogos en orden respectivo) ACF, CBF, ABC. Por ser semejantes, las áreas de los triángulos son proporcionales a los cuadrados de las longitudes de lados homólogos ${ }^{1}$. Por lo tanto, tomando como lados homólogos las hipotenusas e indicando con $a P Q R$ el área del triángulo de vértices $P, Q, R$, se tiene

$$
a \mathrm{ABC} / \mathrm{AB}^{2}=\boldsymbol{a} \mathrm{ACF} / \mathrm{AC}^{2}=\boldsymbol{a} \mathrm{CBF} / \mathrm{CB}^{2}=k
$$

de donde

$$
a \mathrm{ABC}=k \mathrm{AB}^{2}, a \mathrm{ACF}=k \mathrm{AC}^{2}, a \mathrm{CBF}=k \mathrm{CB}^{2}
$$

Es evidente que el área del triángulo mayor es la suma de las áreas de los triángulos menores, es decir

$$
a \mathrm{ABC}=\boldsymbol{a} \mathrm{ACF}+\boldsymbol{a} \mathrm{CBF}
$$

combinando las últimas dos igualdades se obtiene

$$
k A B^{2}=k A C^{2}+k C B^{2}
$$

Preferimos dejar la última expresión con el factor $k$ multiplicando cada uno de los términos, aunque obviamente se podría simplificar, porque aquí es donde la demostración inspira la generalización: Si construimos sobre los lados del triángulo rectángulo original ABC figuras semejantes ${ }^{2}$, sus áreas estarán en relación con los cuadrados de las distancias entre pares de puntos homólogos. Si tomamos como puntos homólogos los extremos de los lados del triángulo rectángulo $\mathrm{ABC}$, entonces se cumple una ecuación igual a la ecuación (\#), (con una $\mathrm{k}$ posiblemente diferente pero igual para los tres términos) y que por lo tanto se puede cancelar. El Teorema extendido de Pitágoras queda entonces como sigue:

\section{Teorema extendido de Pitágoras}

"El área de una figura cualquiera sobre la hipotenusa de un triángulo rectángulo es igual a la suma de las áreas de figuras semejantes sobre sus catetos."

Aquí se entenderá por "cualquiera" las figuras como las que aparecen en los libros de geometría, las cuales incluyen polígonos regulares e irregulares, así como figuras cuyas fronteras sean curvas, no necesariamente simples, (en cuyo caso interesa el sentido de circulación en la frontera y hay

\footnotetext{
Se usa el teorema: "si dos triángulos son semejantes, la relación de sus áreas es el cuadrado de la relación de dos lados correspondientes". (Moise, 1968)

2 Informalmente son figuras semejantes las que se obtienen al fotografiar una figura geométrica y hacer una ampliación o reducción fotográfica. También son semejantes los mapas dibujados a diferentes escalas.
} 
áreas positivas y negativas) y no necesariamente conectadas (pero en número finito) que tienen, por pedazos, fronteras con un número finito de funciones analíticas como las cuadráticas (pedazos de círculo, parábola, elipse, hipérbola), polinomios, splines, senoides, exponenciales, etc.), que estén cerradas y que tengan bien definido el concepto de área encerrada por la curva o curvas. El autor está dispuesto a sacrificar casos cuestionables como los que gustan inventar los matemáticos o, que se dice, aparecen en la naturaleza como ciertos fractales con perímetros infinitos y áreas difíciles o imposibles de calcular, o de las encerradas en funciones que tienen un cierto valor en los puntos racionales y diferente valor en los puntos irracionales, pues no se tiene la intención de entrar a la geometría diferencial, a la topología, la integración de Lebesgue o de la teoría de la medida para este asunto. Restringiremos a las figuras semejantes para las cuales los matemáticos hayan demostrado que sus áreas son proporcionales a los cuadrados de las distancias entre puntos homólogos. El autor ha visto en libros elementales teoremas que lo aseguran para polígonos simples (Jurgensen et al., 1963). De allí en adelante, y aún para el caso de polígonos en cierta medida, los matemáticos se comienzan a meter en honduras que los obligan a salir de la geometría elemental y entrar al análisis y la geometría diferencial o topología.

\section{Ilustración del Teorema extendido de Pitágoras}

Vamos a verificar con un caso numérico la aplicación del Teorema extendido de Pitágoras. Utilizaremos el clásico triángulo rectángulo cuyos catetos valen 3, 4 y cuya hipotenusa vale 5. Escogeremos una forma muy sencilla para no tener que trabajar mucho. La forma se muestra en la figura 6.

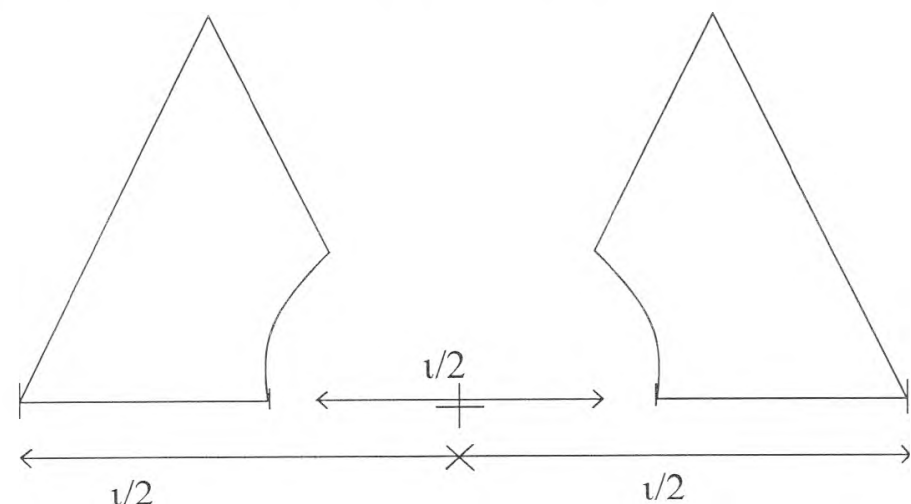

Figura 6

En la figura 6 se muestra una forma con longitud base $Z$. La base está dividida en dos porciones, cada una ocupa la mitad. Cada mitad tiene un triángulo equilátero de lado igual a $\ell / 2$. Con el centro señalado por una crucecita con un hueco circular se elimina un pedazo de cada triángulo con forma de sector circular. El círculo tiene un diámetro cuya longitud es $l / 2$. Si figuras semejantes son colocadas a las escalas que les corresponden para las longitudes de los lados del triángulo rectángulo con medidas 3,4 y 5 se obtienen los resultados que se muestran en la figura 7 . Este es un caso en el que las fronteras de la figura no están conectadas y tienen curvas.

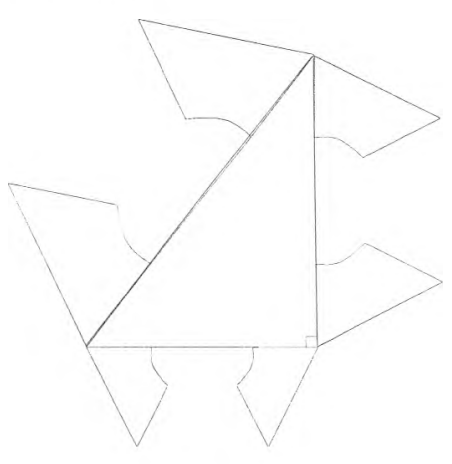

Figura 7

Los pares de triángulos (incompletos) apoyados sobre la hipotenusa y los catetos mayor y menor del triángulo de la figura 7 , tienen como base segmentos de recta con longitudes $2.5,2.0$, y 1.5 , respectivamente, y alturas $2.5 \cos 30^{\circ}$, $2.0 \cos 30^{\circ}$, y $1.5 \cos 30^{\circ}$, respectivamente. Ignorando por el momento, el agujero circular, las áreas serían respectivamente para la hipotenusa, cateto mayor y cateto menor: $2.5^{2} \cos 30^{\circ}, 2.0^{2} \cos 30^{\circ}, 1.5^{2} \cos 30^{\circ}$. A esto hay que restar al área asociada con cada lado, dos sectores circulares de 60 grados de círculos con diámetros 2.5, 2.0, y 1.5 que valen $(1 / 12) \pi 2.5^{2},(1 / 12) \pi 2.0^{2},(1 / 12) \pi 1.5^{2}$, respectivamente. Así, las áreas totales correspondientes a la hipotenusa y los dos catetos son:

$$
\begin{aligned}
& \text { Hipotenusa: } 2.5^{2}(\cos 30-\pi / 12)=1 / 4 \times 25 \times 0.6042= \\
& 3.7763 \\
& \text { Cateto Mayor: } 2.0^{2}(\cos 30-\pi / 12)=1 / 4 \times 16 \times 0.6042= \\
& 2.4168 \\
& \text { Cateto Menor: } 1.5^{2}(\cos 30-\pi 12)=1 / 4 \times 9 \times 0.6042= \\
& 1.3595
\end{aligned}
$$

La revisión de la suma de las áreas de las figuras sobre los catetos arroja

$$
1.3595+2.4168=3.7763
$$

Por lo tanto, el teorema se cumple.

En la última lista de áreas es muy evidente la influencia del teorema que dice que las figuras semejantes tienen áreas proporcionales a los cuadrados de las distancias entre puntos homólogos. Aquí las distancias utilizadas fueron las longitudes de los lados del triángulo rectángulo 5, 4 y 3. El 
factor de proporcionalidad común en nuestro ejemplo es $0.6042 / 4=0.15105$. Queda claro entonces que para verificar el teorema no es necesario hacer todos los cálculos en detalle, si se hace con cuidado debe salir como factor común en las tres áreas el factor de proporcionalidad multiplicado por los cuadrados de los lados. Mientras los lados del triángulo rectángulo satisfagan el Teorema de Pitágoras lo demás está asegurado.

\section{Discusión}

No tenemos duda que todos los geómetras competentes contestarían que es evidente la veracidad del Teorema extendido de Pitágoras presentado aquí. Sin embargo, tras una pequeña encuesta personal entre colegas ingenieros y de otras profesiones (físicos, biólogos, actuarios, geógrafos, computólogos y hasta matemáticos, muchos de ellos profesores universitarios fue convincente que casi nadie estaba consciente del hecho y varios mostraron sorpresa de que el teorema fuera cierto y tan general. El espíritu del Teorema de Pitágoras como lo entendían los griegos antiguos, de acuerdo con Moise y Downs (1975), es muy cercano al enfoque en este artículo. La mayor parte de la larga demostración que da Euclides en los Elementos está orientada a establecer la igualdad entre las áreas de los cuadrados construidos sobre los catetos y los rectángulos que sumados resultan el área del cuadrado construido sobre la hipotenusa. Si en vez de cuadrados se hubieran construido figuras como las que se utilizaron para hacer la verificación numérica, la demostración hubiera sido verdaderamente laboriosa (sobre todo por la forma circular). El secreto de la eficiencia de la demostración en este artículo es usar el teorema sobre figuras semejantes y cuadrados de distancias entre puntos homólogos. Este teorema, importante por central en geometría, nos permite cubrir mucha distancia en pocos pasos. Cuando se quiere resulta parecido el viajar por avión para trasladarse a otro continente, no hay que resolver el problema de determinar la mejor ruta para viajar a un puerto cercano y luego de viajar a un puerto cercano al destino, para finalmente viajar de allí a nuestro destino. Por avión, si las ciudades son grandes, lo probable es que haya un vuelo directo entre origen y destino.

\section{Generalización del Teorema de Varignon}

En muchos libros de geometría (Coxeter, 1971), (Moise y Downs, 1975) y ( Yaglom, 1962) se menciona el siguiente teorema debido a Pierre Varignon (1654-1722):

"Sobre un cuadrilátero simple cualquiera, si se unen los puntos medios de los lados adyacentes, la figura resultante es un paralelogramo. Las líneas que unen los puntos medios de los lados opuestos (llamados bimedianas del cuadrilátero original) se bisecan".

La demostración del teorema es simple, tanto por métodos geométricos elementales, como utilizando números complejos (Murray-Lasso, 2000). El autor considera la demostración utilizando números complejos potencialmente más poderosos, pues se presta más para demostrar extensiones del teorema, como se verá más adelante.

Demostración del Teorema de Varignon por métodos geométricos elementales

Sea el cuadrilátero con vértices $A B C D$ y los puntos $P, Q, R$, $\mathrm{S}$ los puntos medios de los lados del cuadrilátero que a su vez son los vértices de un segundo cuadrilátero, como se muestra en la figura 8 . Trácense con líneas punteadas las diagonales $\mathrm{AC}$ y $\mathrm{BD}$ del cuadrilátero original. Se forman varios triángulos. Concentrémonos en los triángulos $\nabla A C D$ y $\nabla$ SRD. Dichos triángulos son semejantes por el teorema "lado - ángulo - lado" de los triángulos semejantes y los segmentos SR y AC son paralelos, siendo la longitud del primero la mitad de la longitud del segundo. Por igual razón los triángulos $\nabla \mathrm{ACB}$ y $\nabla \mathrm{PQB}$ son semejantes y los segmentos $P Q$ y AC paralelos, y la longitud del primero igual a la mitad de la longitud del segundo. Por transitividad deducimos entonces que los segmentos SR y PQ tienen igual longitud y son paralelos.

Si ahora nos fijamos en los triángulos que se forman con la diagonal $\mathrm{DB}$, con iguales argumentos podemos concluir que los segmentos RQ y SP tienen igual longitud y son paralelos. Como el cuadrilátero que une los puntos medios PQRS tiene lados opuestos paralelos y de iguales longitudes (consecuencia de que ambos pares sean paralelos), concluimos que se trata de un paralelogramo. Finalmente, las bimedianas del cuadrilátero original son las diagonales del paralelogramo, éstas, por un teorema conocido se bisecan (Jurgensen, et al.,1963) situación que se puede demostrar fácilmente ya sea por consideraciones de simetría o porque se forman pares de triángulos semejantes $\nabla$ SRT y $\nabla$ PQT; $\nabla R Q T$ y $\nabla$ PST.

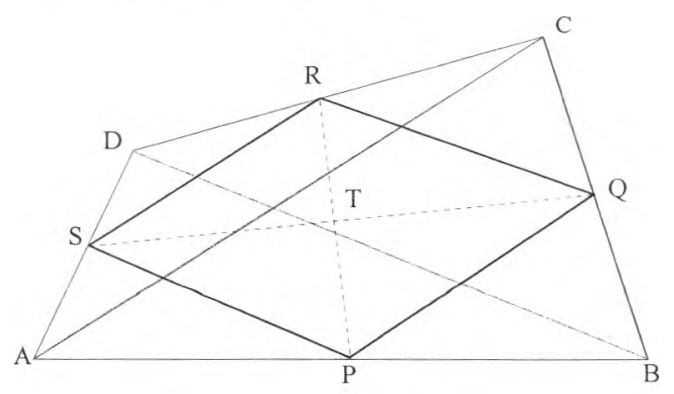

Figura 8 
Demostración del Teorema de Varignon con números complejos

En la figura 9 asociamos los números complejos $2 z_{1}, 2 z_{2}, 2 z_{3}$ y $2 z_{4}$ con los lados del cuadrilátero original. Tomemos el origen en el punto $\mathrm{O}$. Los lados del cuadrilátero que une

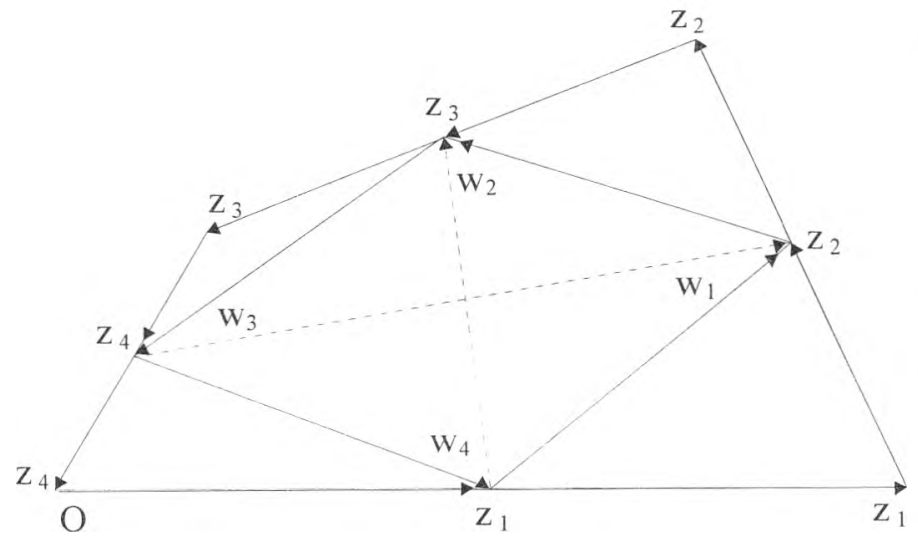

Figura 9

los puntos medios de los lados del cuadrilátero original los asociamos con los números complejos $w_{1}, w_{2}, w_{3}, w_{4}$ como se muestra en la figura 9. Debido a que el polígono del cuadrilátero original es cerrado se tiene

$$
\begin{gathered}
2\left(z_{1}+z_{2}+z_{3}+z_{4}\right)=0, \quad \text { de donde } z_{1}+z_{2}= \\
-\left(z_{3}+z_{4}\right), z_{2}+z^{3}=-\left(z_{1}+z_{4}\right)
\end{gathered}
$$

Por inspección de la figura 9 se tiene:

$$
\begin{aligned}
w_{1}=z_{1}+z_{2}, & w_{2}=z_{2}+z_{3}, \\
w_{3}=z_{3}+z_{4}, & w_{4}=z_{4}+z_{1}
\end{aligned}
$$

y utilizando la ecuación (1) nos queda

$$
w_{1}=-w_{3}, w_{2}=-w_{4}
$$

Las igualdades (3) nos indican que los lados opuestos del cuadrilátero que une los puntos medios de los lados del cuadrilátero original son paralelos y de igual longitud, por lo tanto forman un paralelogramo.

El punto medio del segmento que va del centro del lado inferior al centro del lado superior es

$$
\begin{gathered}
\mathrm{C}=z_{1}+1 / 2\left(w_{1}+w_{2}\right)=z_{1}+0.5\left(z_{1}+2 z_{2}+z_{3}\right)= \\
1.5 z_{1}+z_{2}+0.5 z_{3}
\end{gathered}
$$

Donde se tomó en consideración la ecuación (2). A su vez, el punto medio del segmento que va del centro del lado izquierdo al centro del lado derecho es

$$
\begin{gathered}
\mathrm{D}=-z_{4}+1 / 2\left(w_{4}+w_{1}\right)= \\
z_{1}+z_{2}+z_{3}+1 / 2\left(-z_{2}-z_{3}+z_{1}+z_{2}\right)= \\
1.5 z_{1}+z_{2}+0.5 z_{3}
\end{gathered}
$$

Tomando en consideración las ecuaciones (1) y (2). En vista que las expresiones de $C$ y $D$ son idénticas, independientemente de los valores de las z's, los puntos $\mathrm{C} y$ $D$ coinciden, o lo que es lo mismo, las bimedianas se bisecan.

\section{Extensiones del Teorema de Varignon}

Existen algunos teoremas que se pueden considerar como extensiones del Teorema de Varignon, por ejemplo, en Yaglom (1962) aparece lo siguiente: "En los lados de un cuadrilátero arbitrario (convexo) $\mathrm{ABCD}$ se construyen triángulos equiláteros $\mathrm{ABM}_{1}, \mathrm{BCM}_{2}, \mathrm{CDM}_{3}$ y $\mathrm{DAM}_{4}$ en forma tal, que el segundo y el cuarto son exteriores al cuadrilátero y el primero y tercero están del mismo lado relativos a los lados $A B$ y $\mathrm{CD}$ que el propio cuadrilátero. El cuadrilátero $\mathrm{M}_{1} \mathrm{M}_{2} \mathrm{M}_{3} \mathrm{M}_{4}$ es un paralelogramo (Figura 10). En casos especiales el paralelogramo puede degenerar en un intervalo."

Vamos a demostrar este teorema usando números complejos para ilustrar una de las estrategias para encontrar extensiones a teoremas de este tipo.

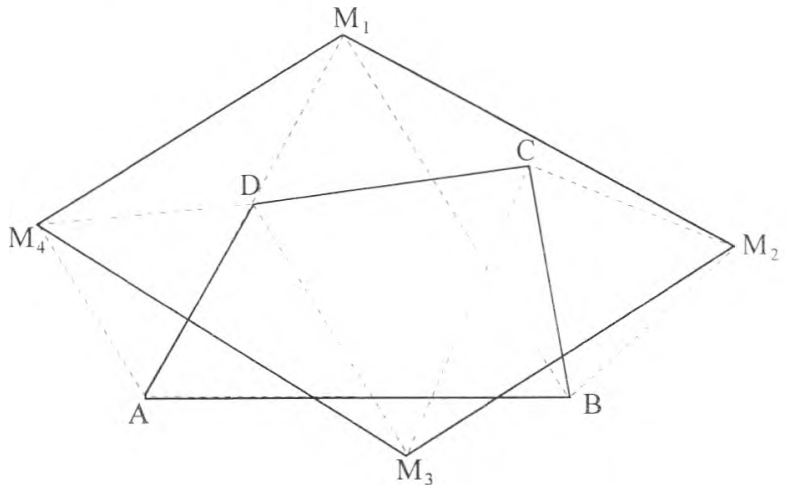

Figura 10

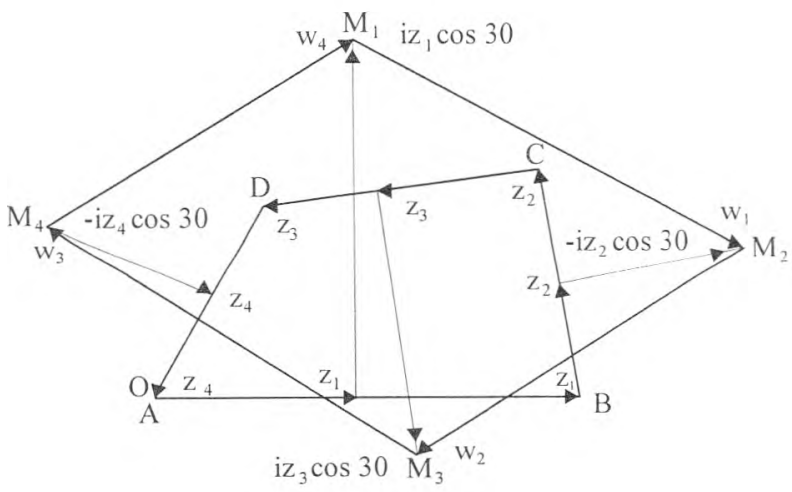

Figura 11 
En la figura 11 se ha colocado el origen del plano complejo en el punto A. Los lados del cuadrilátero original serán los números complejos $2 z_{1}, 2 z_{2}, 2 z_{3}$ y $2 z_{4}$ (usamos los factores 2 para localizar con facilidad los puntos medios de los lados). Como solamente interesan los vértices extremos de los triángulos equiláteros, se puede sustituir cada triángulo por un número complejo que parte del centro del lado donde se apoya el triángulo y es ortogonal al mismo, orientado hacia la izquierda al recorrer los lados 1 y 3 y hacia la derecha al recorrer los lados 2 y 4 . Las longitudes de los números complejos que representan los triángulos equiláteros son las alturas de los triángulos equiláteros que valen $\left|v_{i}\right| \cos 30$. Los que van a la derecha se les multiplica por - i y los que van a la izquierda por $i$ (recordar que al multiplicar un número complejo por $i$ lo rota 90 grados en el sentido positivo, es decir, contrario a las manecillas del reloj).

De la figura 11 obtenemos por inspección que

$$
\begin{gathered}
2 z_{1}+2 z_{2}+2 z_{3}+2 z_{4}=0 \text { o lo que es lo mismo } \\
z_{1}+z_{2}=-z_{3}-z_{4}, z_{2}+z_{3}=-z_{1}-z_{4}
\end{gathered}
$$

los números complejos que representan los lados del cuadrilátero que tiene por vértices los puntos $M_{1}, M_{2}, M_{3}$ y $\mathrm{M}_{4}$ son

$$
\begin{aligned}
\left(\mathrm{M}_{1} \mathrm{M}_{2}\right): w_{1}=- & i z_{1} \cos 30+z_{1}+z_{2}-i z_{2} \cos 30=z_{1}+ \\
& z_{2}-(i \cos 30)\left(z_{1}+z_{2}\right) \\
\left(\mathrm{M}_{2} \mathrm{M}_{3}\right): w_{2}= & i z_{2} \cos 30+z_{2}+z_{3}+i z_{3} \cos 30=z_{2}+ \\
& z_{3}+(i \cos 30)\left(z_{2}+z_{3}\right) \\
\left(\mathrm{M}_{3} \mathrm{M}_{4}\right): w_{3}=- & -i z_{3} \cos 30+z_{3}+z_{4}-i z_{4} \cos 30=-z_{1} \\
& -z_{2}+(i \cos 30)\left(z_{1}+z_{2}\right) \\
\left(\mathrm{M}_{4} \mathrm{M}_{1}\right): w_{4}= & i z_{4} \cos 30+z_{4}+z_{1}+i z_{1} \cos 30=-z_{2}- \\
& z_{3}-(i \cos 30)\left(z_{2}+z_{3}\right)
\end{aligned}
$$

De las últimas ecuaciones está claro que $w_{1}=-w_{3} \mathrm{y}$ $w_{2}=-w_{4}$, que significa que las longitudes de $w_{1}$ y $w_{3}$ son iguales y que las longitudes de $w_{2}$ y $w_{4}$ son iguales. En otras palabras, la figura cuyos vértices son $M_{1}, M_{2}, M_{3}$ y $M_{4}$ es un paralelogramo.

La extensión del teorema de los triángulos equiláteros apoyados sobre los lados del cuadrilátero proviene de dos observaciones:

1) Podemos sustituir cos 30 por cualquier número real (positivo o negativo) y las conclusiones siguen siendo las mismas, es decir, los puntos $M_{1}, M_{2}, M_{3}$, y $M_{4}$ siguen siendo vértices de un paralelogramo.
2) Podemos sustituir $(i \cos 30)$ por cualquier número complejo (el cual representa un operador que actúa sobre los números complejos $z_{i}$, y-nuevamente las conclusiones son que $M_{1}, M_{2}, M_{3}$ y $M_{4}$ son los vértices de un paralelogramo.

Las consecuencias de lo anterior nos permite generar dos nuevas familias de teoremas que se podrían enunciar como sigue:

\section{Teorema 1:}

Si sobre un cuadrilátero arbitrario recorrido en orden de vértices contiguos se construyen figuras semejantes con simetría axial relativas a una línea perpendicular al lado sobre el que se construyen, que pase por el centro de dicho lado, alternándose unas a la izquierda y otras a la derecha, los puntos homólogos situados sobre los ejes de simetría son los vértices de un paralelogramo.

\section{Teorema 2:}

Si sobre un cuadrilátero arbitrario recorrido en orden de vértices contiguos se construyen figuras semejantes arbitrarias sobre los lados 1 y 3 y sobre los lados 2 y 4 se construyen las reflexiones de dichas figuras con respecto al centro de los lados en las que se apoyan las figuras, los puntos homólogos en cualquier parte de la figura son los vértices de un paralelogramo.

Para ilustrar el teorema 1 escogemos como figuras con simetría axial semicírculos y escogemos como puntos homólogos sobre los ejes de simetría axial los puntos a la mitad del radio perpendicular a la base de un semicírculo. La figura 12 muestra la situación.

Cabe señalar que si tomamos como puntos homólogos en la figura 12 los centros de los círculos, el paralelogramo que se forma es el del Teorema de Varignon, por lo que dicho teorema se puede considerar como un caso particular del teorema 1 de este artículo.

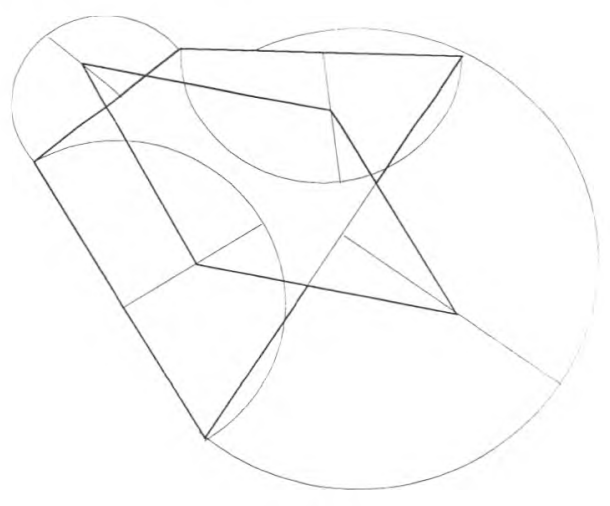

Figura 12 


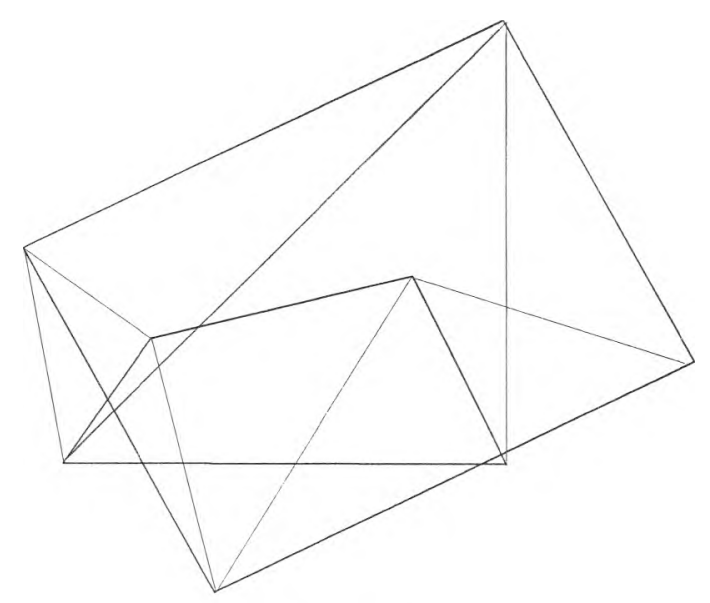

Figura 13

Para ilustrar el teorema 2 construiremos sobre los lados de un cuadrilátero triángulos rectángulos con ángulos agudos de 45 grados orientados según el teorema.

En la figura 13 los triángulos rectángulos exteriores (lados derecho e izquierdo del cuadrilátero) tienen su hipotenusa hacia el frente, si es que los triángulos viajaran en sentido contra horario recorriendo los vértices del cuadrilátero original. En los lados superior e inferior en el que están dibujados hacia adentro del cuadrilátero original uno de los catetos es el que va al frente. En todos los casos, uno de los catetos es la base y se hace coincidir con el lado sobre el cual se apoya el triángulo rectángulo. Tomamos como puntos homólogos los vértices más alejados de la base siendo ésos los vértices del paralelogramo. Aunque hemos dibujado cuadriláteros convexos, por la manera en que se hizo la demostración, no existe tal restricción. Además de cuadriláteros no convexos las figuras pueden ser tales que sus lados se cruzan como se muestra en la figura 14 .

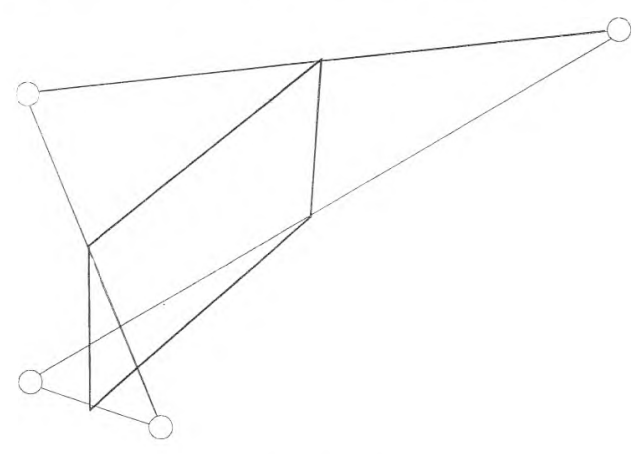

Figura 14

En la figura 14 los extremos de los lados están marcados con pequeños círculos. El cruce sin círculo no indica vértice de la figura. En la figura se muestra el paralelogramo del Teorema de Varignon, el cual también se cumple para figuras que se cruzan. La demostración por medio de números complejos contempla esta situación, ya que los números complejos que se usan son arbitrarios con el único requisito que cada lado parta del final del lado anterior y que la figura se cierre al final con el vértice inicial.

\section{La computadora para verificaciones geométricas como apoyo en la enseñanza}

Desde el punto de vista matemático un teorema que ha sido demostrado no requiere que se verifique en casos particulares. Sin embargo, desde el punto de vista didáctico es conveniente que los estudiantes verifiquen los teoremas en algunos casos particulares porque les ayuda a familiarizarse con ellos, a recordar el teorema y analizar las hipótesis supuestas.

Logo es un lenguaje diseñado para propósitos educativos por investigadores del área de la Inteligencia Artificial (Papert, 1980 y 1993). Cuenta con una tortuga que al desplazarse es capaz de dibujar líneas de diferentes colores. Una de las versiones de Logo, LogoWriter (Logo Computer Systems, 1990) además de contar con instrucciones para mover la tortuga hacia delante, atrás, giro a la derecha e izquierda, levantar y bajar la pluma, y los operadores aritméticos, así como las funciones trigonométricas, entre otras, cuenta además con algunas herramientas como una función distancia que sin mayor esfuerzo calcula la distancia que hay del centro de la tortuga (donde tiene la pluma) a un punto especificado como parámetro, la función hacia que regresa el rumbo de la línea que hay entre la tortuga y un punto especificado como parámetro, las funciones coorx y coory que regresan las coordenadas $x$ e $y$ de la tortuga.

A continuación mostramos el listado de un procedimiento para verificar el Teorema de Varignon y las dos extensiones presentadas en este artículo.

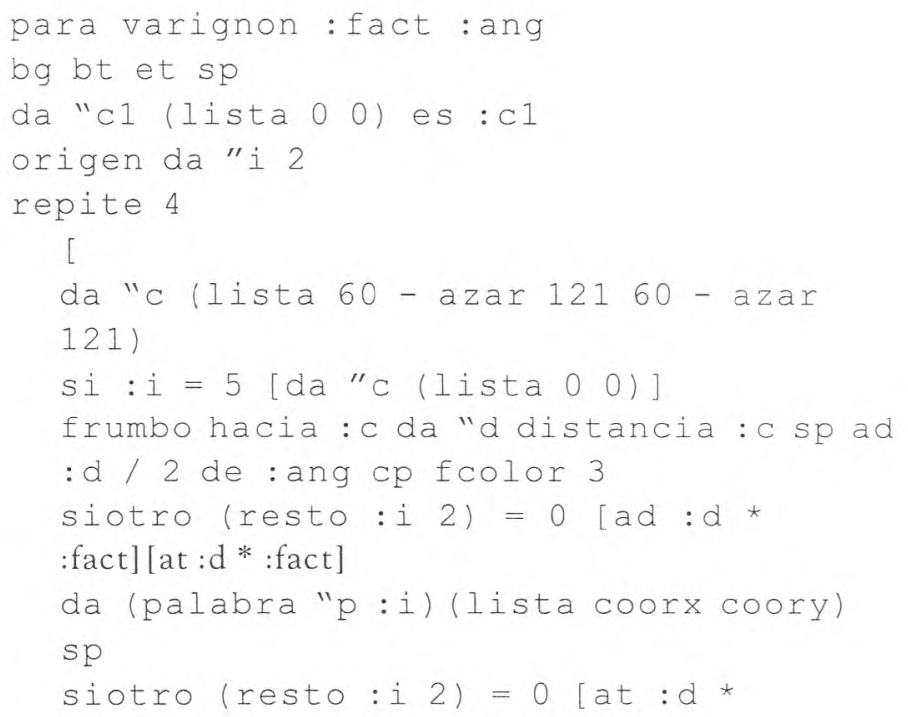




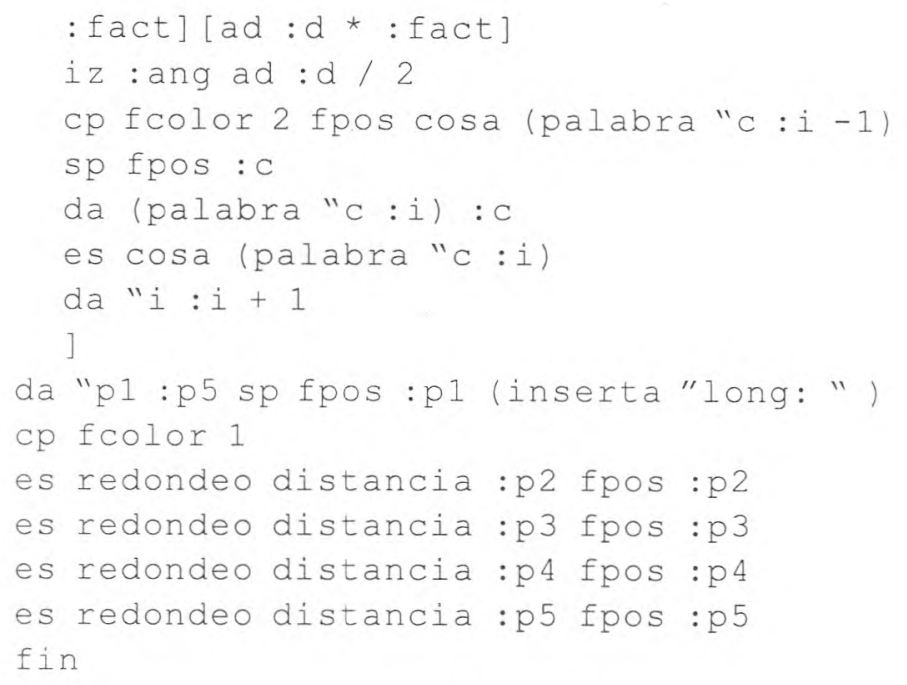

La explicación del programa es la siguiente:

El procedimiento tiene dos parámetros: fact y ang. El parámetro fact corresponde a un factor que multiplica a la longitud del lado del cuadrilátero y representa la longitud del número complejo que parte del centro del lado y cuya punta será uno de los vértices del paralelogramo. El parámetro ang corresponde al ángulo del número complejo que va del centro del lado del cuadrilátero al vértice del paralelogramo. Para los lados pares el vector lo dibuja hacia la derecha y para los nones lo gira 180 grados (para simular llegar al punto homólogo de una figura invertida con centro en el punto medio del lado).

El procedimiento comienza por borrar la pantalla de gráficos y texto, esconder la tortuga y subir la pluma de la tortuga. A continuación, escoge el origen del plano complejo en el primer vértice del cuadrilátero y como escribe las coordenadas de los cuatro vértices del cuadrilátero, escribe 00 en el área de trabajo. Manda a la tortuga al origen y comienza una iteración de 4 ciclos que arranca con un valor de 2 para el contador. En cada ciclo genera un par de números aleatorios enteros uniformemente distribuidos entre -60 y 60 que se los asigna a los vértices del cuadrilátero [como el quinto vértice es nuevamente el primer vértice, asigna al quinto vértice el par $(0,0)]$. Generado el par correspondiente al siguiente vértice, la tortuga, que está posicionada en el vértice anterior, se orienta hacia el nuevo vértice y calcula la distancia de su posición a dicho vértice y con la pluma levantada para no dibujar avanza la mitad de dicha distancia (colocándose en el punto medio del lado). De ahí gira hacia la derecha el ángulo ang, baja la pluma y selecciona el color 3. Ahora, si el lado en el cual va es par, avanza una distancia igual a fact multiplicado por la longitud del lado en el que va. (Si el lado es impar, en lugar de avanzar se echa en reversa). Una vez que llega al vértice del paralelogramo guarda sus coordenadas en la componente $i$ del vector $p$. Ahora la tortuga sube la pluma para no dibujar mientras se desplaza y regresa al centro del lado, se orienta en la dirección adecuada para completar la segunda mitad de su trayectoria sin dibujar sobre el lado y lo completa. A continuación, con la pluma baja y en color 2 hace un viaje de ida y vuelta al vértice anterior dibujando el lado en la primera mitad de la trayectoria. Finalmente, guarda en la componente $i$ de cy despliega en el área de trabajo las coordenadas del vértice en el que termina el ciclo. Antes de pasar al siguiente ciclo incrementa el contador $i$ en uno.

Ya fuera de la iteración, como el vector $p(i)$ de coordenadas de los vértices lo llenó de 2 a 5 , copia en p (1) el valor de $p(5)$. Sin pluma, la tortuga se posiciona en $p(1)$ y luego con pluma y color 1 se va posicionando sucesivamente en $p(2), p(3), p(4)$ y $p(5)$ dibujando mientras lo hace, trazando así el paralelogramo. Cada vez que se posiciona, calcula y despliega la distancia al siguiente punto, de esta manera el usuario puede verificar que la figura realmente es un paralelogramo.

Con el procedimiento exhibido se puede verificar tanto el Teorema de Varignon como los teoremas 1 y 2 del artículo. Para el Teorema de Varignon los parámetros deben ser 0 y 0 . (Realmente el segundo parámetro puede ser cualquier cosa pues es un vector de longitud cero). Para el teorema 1 , el segundo argumento debe ser $90^{\circ}$ pues los puntos homólogos deben estar sobre un eje perpendicular al lado que pase por el centro del lado. El primer argumento puede ser cualquier número real (positivo o negativo - si el número se escoge con magnitud demasiado grande la figura se saldrá de la pantalla). Para el teorema 2, el primer argumento puede ser un número real positivo o negativo y el segundo argumento un ángulo entre -180 y 180 grados.

A continuación se exhiben muestras de lo producido en tres corridas del programa varignon. En la figura 15 se muestra una corrida con el teorema de Varignon original. Lo que se teclea es

varignon 00

00 2140

$48-51$

$\begin{array}{rrr}-57 & 27\end{array}$

00

long:51

35

51

35

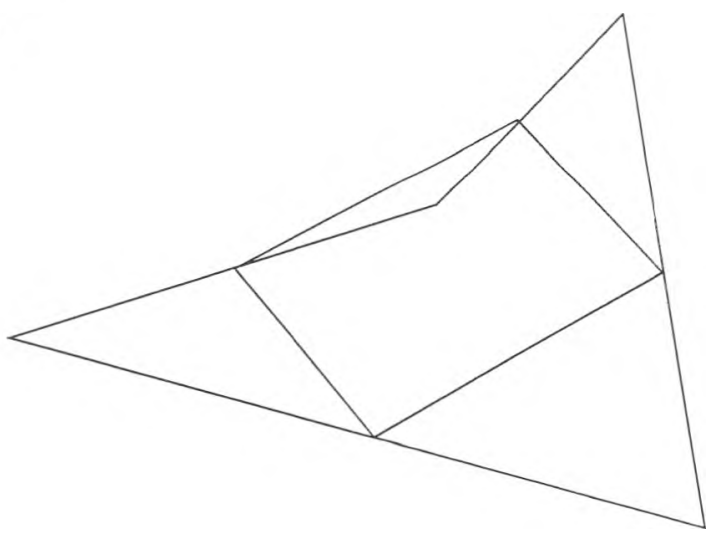

Figura 15 
Los primeros cinco pares de puntos en el área de trabajo de la figura 15 son las coordenadas de los vértices del cuadrilátero (el quinto punto es el primero repetido). Después de la palabra long: los cuatro números son las longitudes de los lados del paralelogramo.

Para probar el Teorema 1 utilizaremos cuadrados, tomando como puntos homólogos los centros de los cuadrados. Así, los vectores estarán a 90 grados de los lados y tendrán longitud igual a la mitad del lado, se teclea
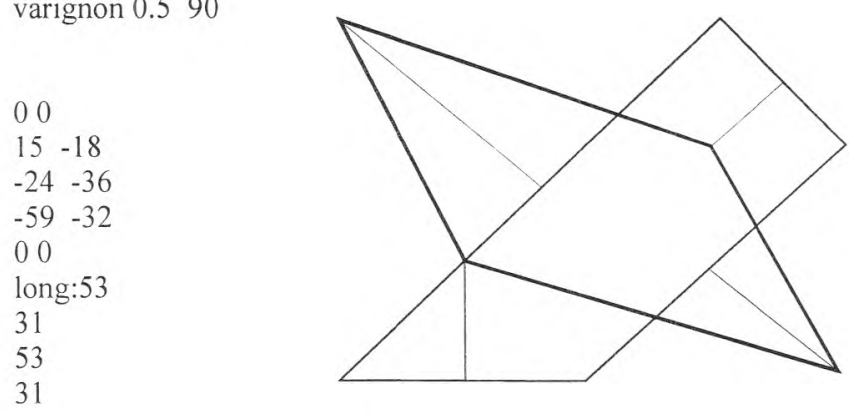

Figura 16

El lector debe imaginar en la figura 16 los cuadrados construidos sobre los lados del cuadrilátero (unos hacia fuera y otros hacia adentro del cuadrilátero). Las líneas delgadas son los números complejos que van de los centros de los lados a los centros de los cuadrados imaginarios. Dichos centros son los vértices del paralelogramo trazado con líneas gruesas. Aunque el programa dibuja el cuadrilátero, el paralelogramo y las líneas de los números complejos que van del centro de los lados a los centros de los cuadrados (imaginarios) de diferentes colores, para presentarlos en blanco y negro se han dibujado los paralelogramos más gruesos, los cuadriláteros un poco menos gruesos y los números complejos para localizar los centros de los cuadrados (imaginarios) delgados.

Paraverificar el teorema 2 , nos imaginamos que se construye sobre cada lado recorrido un triángulo como se muestra en la figura 17. Los triángulos deben tener la misma orientación cuando se recorren los vértices adyacentes del cuadrilátero en un orden específico.

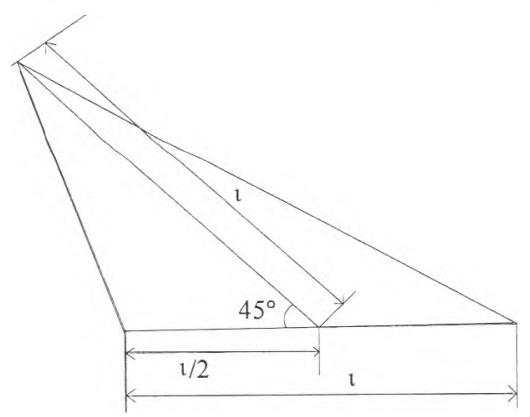

Figura 17
No se tienen que dibujar los triángulos completos, basta colocar el segmento de recta con la inclinación y longitud adecuada para localizar el vértice para determinar el paralelogramo. El factor fact que multiplica al lado del cuadrilátero es la unidad y el ángulo ang es 45 grados. Para ejecutar el programa se teclea

varignon 145
00
$3-58$
$24-37$
$44 \quad 16$
00
long: 118
32
118
32

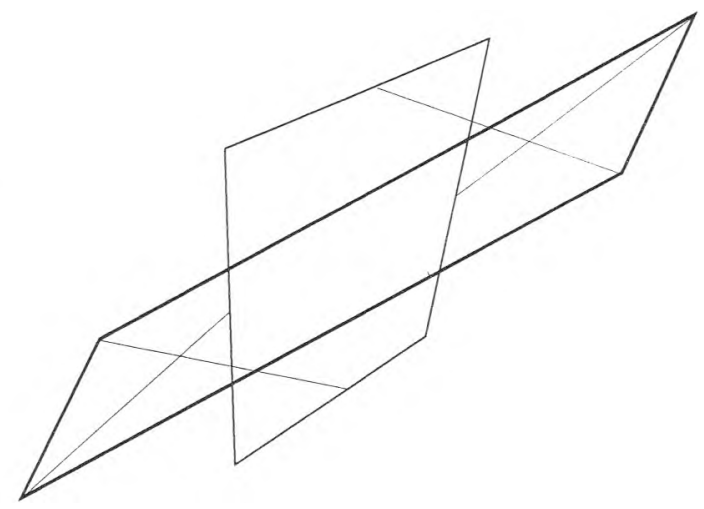

Figura 18

\section{Extensión del Teorema de Varignon en tres dimensiones}

El teorema de Varignon también se cumple en tres dimensiones. Esto se puede ver porque en la demostración con números complejos para el plano, los números complejos solamente se utilizaron en su papel de vectores bidimensionales para la suma y resta. Repetimos la demostración pensando en vectores de tres dimensiones razonando sobre la figura tridimensional 19.

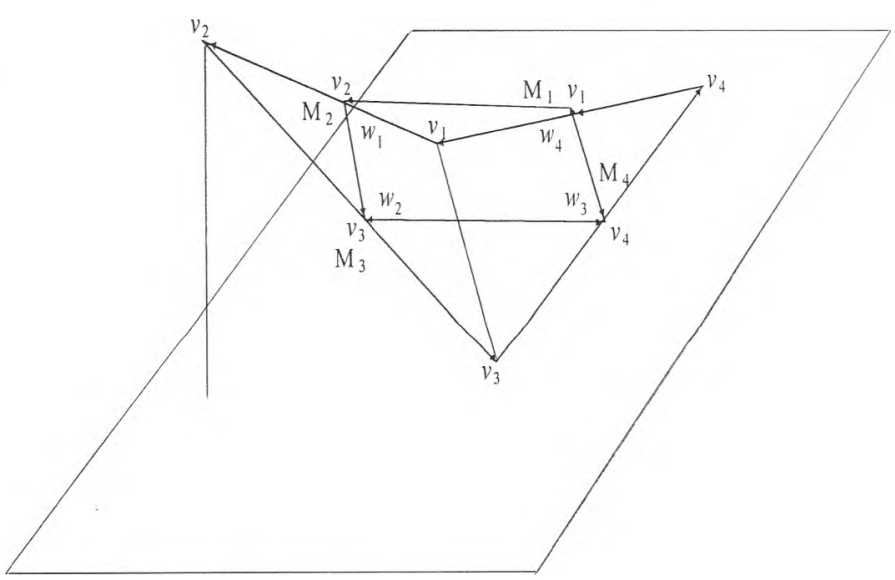


En la figura 19 se muestra un cuadrilátero en el espacio tridimensional, la diagonal trazada con una línea delgada, así como los vectores $v_{1}$ y $v_{4}$, están sobre el plano dibujado. El vértice incidente con una línea vertical punteada está a cierta distancia sobre el plano. La figura 19 sugiere una demostración geométrica elemental del Teorema de Varignon en el espacio. Usando la diagonal con línea delgada, en el plano dibujado, se forman dos triángulos semejantes (lado - lado - lado) con un factor de $1 / 2$ de proporcionalidad entre los lados, por lo tanto, los ángulos son congruentes y el lado correspondiente al vector $w_{4}$ es paralelo a la diagonal delgada. Por la misma razón en el plano levantado del plano, el lado correspondiente al vector $w_{2}$ es paralelo a la diagonal delgada. Por transitividad $w_{4}$ y $w_{2}$ son paralelos. Si se traza la otra diagonal del cuadrilátero original, con el mismo argumento, en otro plano, se puede establecer que los lados $w_{1}$ y $w_{3}$ son paralelos.

Procedemos ahora con una demostración vectorial. Cada medio lado del cuadrilátero está representado por un vector tridimensional $v_{i}, i=1,2,3,4$. Las líneas entre los puntos medios quedan representadas por vectores $w_{i}, i=1$, 2, 3, 4. Debido a que el cuadrilátero se cierra sobre si mismo se tiene

$$
\begin{gathered}
2\left(v_{1}+v_{2}+v_{3}+v_{4}\right)=0, \text { de donde } v_{1}+v_{2}= \\
-\left(v_{3}+v_{4}\right), v_{2}+v_{3}=-\left(v_{1}+v_{4}\right)
\end{gathered}
$$

Por inspección de la figura 19 se tiene:

$$
\begin{aligned}
w_{1}=v_{1}+v_{2}, & w_{2}=v_{2}+v_{3}, \\
w_{3}=v_{3}+v_{4}, & w_{4}=v_{4}+v_{1}
\end{aligned}
$$

y utilizando la ecuación (1') nos queda

$$
w_{1}=-w_{3}, w_{2}=-w_{4}
$$

Notamos que la demostración vectorial es idéntica, excepto por la diferente naturaleza de las z's y las v's (que sin embargo, en cuanto a las operaciones efectuadas obedecen las mismas leyes de manipulación) a la demostración del Teorema de Varignon en el plano. Por lo tanto, sospechamos que hay extensiones del Teorema de Varignon en tres dimensiones parecidas a las demostradas para el plano. Si en el centro de cada lado sumamos para los lados pares y restamos para los impares (o viceversa) un vector obtenido al operar con un operador sobre el vector que representa a medio lado del cuadrilátero, tendríamos las siguientes expresiones para los lados del cuadrilátero que une las puntas $M_{1}, M_{2}, M_{3}$ y $M_{4}$ de los vectores citados.

$$
\begin{gathered}
\left(M_{1} M_{2}\right): w_{1}=-O p v_{1}+v_{1}+v_{2}-O p v_{2}= \\
v_{1}+v_{2}-O p\left(v_{1}+v_{2}\right)
\end{gathered}
$$

$$
\begin{aligned}
\left(\mathrm{M}_{2} \mathrm{M}_{3}\right): & w_{2}=O p v_{2}+v_{2}+v_{3}+O p v_{3}= \\
& v_{2}+v_{3}+O p\left(v_{2}+v_{3}\right) \\
\left(\mathrm{M}_{3} \mathrm{M}_{4}\right): & w_{3}=-O p v_{3}+v_{3}+v_{4}-O p v_{4}= \\
& -v_{1}-v_{2}+O p\left(v_{1}+v_{2}\right) \\
\left(\mathrm{M}_{4} \mathrm{M}_{1}\right): & w_{4}=O p v_{4}+v_{4}+v_{1}+O p v_{1}= \\
& -v_{2}-v_{3}-O p\left(v_{2}+v_{3}\right)
\end{aligned}
$$

De los últimos miembros de estas ecuaciones se obtiene $w_{1}=-w_{3}, w_{2}=-w_{4}$. Lo que significa que los puntos $M_{1}$, $M_{2}, M_{3}$ y $M_{4}$ son los vértices de un paralelogramo (que está en un plano, ya que los vectores paralelos definen ya sea un plano o planos paralelos, pues ambos, por ser paralelos son ortogonales al mismo vector normal al plano y cada trío de puntos $M_{1}, M_{2}, M_{3}$ y $M_{2}, M_{3}, M_{4}$ definen planos, por lo que por transitividad los planos se reducen a uno solo. Naturalmente, se pueden dar casos en que el paralelogramo se degenera en una línea, pues nada impide que se anulen algunos de los vectores $w_{i}$, o que las cuatro $w$ 's sean colineales. Tenemos, sin embargo, el derecho de llamar paralelogramo en un plano a un segmento de recta recorrida dos veces con cuatro vértices en los extremoso dos en los extremos y dos coincidentes entre ellos.

Sólo resta especificar qué cosa es Op. Por la manera en que se manipuló $O p$, se trata de un operador lineal (pues supusimos válida la ley distributiva con respecto a la suma y sacamos signos negativos debajo del operador). Cualquier operador lineal en el espacio vectorial tridimensional puede ser representado por una matriz (Taylor, 1958). Por lo tanto, podemos representar con respecto a bases del espacio tridimensional, al operador Op por medio de una matriz $3 \times 3$. Por ejemplo, podemos utilizar cualquier transformación de reflexión, de rotación o en general cualquier transformación isométrica o cualquier transformación afín (Modenov y Parkhomenko,1965). No es indispensable que la transformación sea no - singular, incluso la transformación cero es aceptable, pues en ese caso el paralelogramo resultante es el del Teorema Original de Varignon.

Una transformación afín es una transformación lineal, por lo que puede ser representada por una matriz $3 \times 3$. Todas las transformaciones lineales nos llevarán a vértices de un paralelogramo, de acuerdo con nuestra demostración algebraica basada en vectores tridimensionales. Podríamos entonces enunciar el nuevo teorema como sigue:

\section{Teorema 3}

Para cualquier cuadrilátero en el espacio de tres dimensiones construido con vectores que lo recorren en un sentido específico, si al vector que representa el punto medio de cada lado par del cuadrilátero le sumamos un vector obtenido al multiplicar una determinada matriz $3 \times 3$ por el vector que 
representa al lado correspondiente y a los puntos medios de los lados impares les restamos el producto de la misma matriz por el vector que representa el lado correspondiente, las puntas de los cuatro vectores resultantes son los vértices de un paralelogramo.

Las transformaciones afines nos dan una gran variedad de posibilidades, ya que son transformaciones del espacio tridimensional que mapea líneas rectas en líneas rectas y planos en planos, conservando el orden de los puntos sobre las líneas, que significa que para cualquier trío de puntos distintos $A, B$ y $C$ sobre cualquier línea recta en el espacio, con $B$ entre $A$ y $C$, las imágenes correspondientes $A^{\prime}, B^{\prime}$ y $C^{\prime}$ estarán sobre una línea recta y $B^{\prime}$ estará entre $A^{\prime}$ y $C^{\prime}$. Otras de las propiedades de las transformaciones afines son que líneas paralelas y planos paralelos se mapean en líneas paralelas y planos paralelos, y líneas y planos que se cruzan se mapean en líneas y planos que se cruzan (Modenov y Parkhomenko, 1965).

\section{Verificación numérica del Teorema de Varignon extendido en el espacio}

Para ilustrar el teorema 3 considérese la figura 20 en la que se muestra el cuadrilátero tridimensional con vértices $A:(0,0,0), B:(0,2,0), C:(0,0,2)$ y $D:(2,0,0)$. Se toma el origen $O$ en $A$. Los centros de los lados son los puntos $P:(0,1,0), Q:(0,1,1), R:(1,0,1)$ y $S:(1,0,0)$.

Por inspección, los vectores $v_{i}$ son: $v_{1}=(0,1,0), v_{2}=(0$, $-1,1), v_{3}=(1,0,-1)$ y $v_{4}=(-1,0,0)$

Tomemos la matriz no singular

$$
\mathcal{O}=\left[\begin{array}{rrr}
1 & 1 & 0 \\
-1 & 1 & 0 \\
0 & 0 & -1
\end{array}\right]
$$

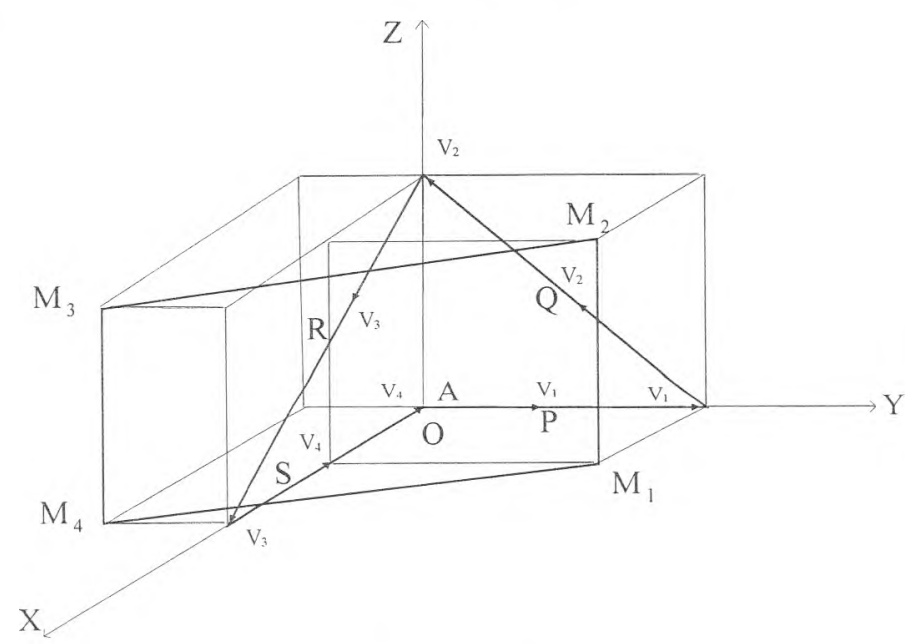

Figura 20
Las posiciones de los puntos $M_{1}, M_{2}, M_{3}$ y $M_{4}$ (que son los vértices del paralelogramo) son:

$$
\begin{aligned}
& M_{1}: P+\mathcal{O} v_{1}=(0,1,0)+(1,1,0)=(1,2,0) \\
& M_{2}: Q-\mathcal{O} v_{2}=(0,1,1)-(-1,-1,-1)=(1,2,2) \\
& M_{3}: R+\mathcal{O} v_{3}=(1,0,1)+(1,-1,1)=(2,-1,2) \\
& M_{4}: S-\mathcal{O} v_{4}=(1,0,0)-(-1,1,0)=(2,-1,0)
\end{aligned}
$$

Si se unen los puntos $M_{i}$, los cuadrados de las longitudes de los lados son:

$$
\begin{aligned}
& d^{2}\left(M_{1}, M_{2}\right)=0+0+2^{2}=4, d^{2}\left(M_{2}, M_{3}\right)=1^{2}+3^{2}+0 \\
& =10, d^{2}\left(M_{3}, M_{4}\right)=0+0+2^{2}=4, \\
& d^{2}\left(M_{4}, M_{1}\right)=1^{2}+3^{2}+0=10
\end{aligned}
$$

Los vectores $w_{i}$ están dados por

$$
\begin{aligned}
& w_{1}=M_{2}-M_{1}=(1,2,2)-(1,2,0)=(0,0,2) \\
& w_{2}=M_{3}-M_{2}=(2,-1,2)-(1,2,2)=(1,-3,0) \\
& w_{3}=M_{4}-M_{3}=(2,-1,0)-(2,-1,2)=(0,0,-2)=-w_{1} \\
& w_{4}=M_{1}-M_{4}=(1,2,0)-(2,-1,0)=(-1,3,0)=-w_{2}
\end{aligned}
$$

Está claro que se trata de un paralelogramo, ya que las longitudes de los lados pares son iguales y los vectores correspondientes son paralelos (uno es negativo del otro) y lo mismo pasa para los lados impares. El paralelogramo se muestra en la figura 20.

Si ahora tomamos otra matriz, podemos calcular diferentes puntos $M_{i}$ que también serán los vértices de un paralelogramo. Tomemos ahora una matriz singular.

$$
\mathcal{O}=\left[\begin{array}{lll}
1 & 1 & 1 \\
1 & 1 & 1 \\
1 & 1 & 1
\end{array}\right]
$$

Las coordenadas de los puntos $P, Q, R, S$ y las componentes de los vectores $v_{1}, v_{2}, v_{3}$ y $v_{4}$ son los previamente calculados. Solamente hay que calcular las componentes de los vectores $\mathrm{O} v_{1}, \mathrm{Ov}_{2}, \mathrm{Ov}_{3}$ y $\mathrm{O} v_{4}$ los cuales son respectivamente: $(1,1,1),(0,0,0),(0,0,0),(-1,-1,-1)$. Las coordenadas de los puntos $M_{i}$ son:

$M_{1}=(1,2,1), M_{2}=(0,1,1), M_{3}=(1,0,1)$ y $M_{4}=(2,1,1)$

Y los vectores $w_{i}$ son:

$$
\begin{aligned}
w_{1}=M_{2}-M_{1} & =(-1,-1,0) ; w_{2}=M_{3}-M_{2}=(1,-1,0) ; \\
w_{3} & =M_{4}-M_{3}=(1,1,0)=-w_{1} ; \\
w_{4} & =M_{1}-M_{4}=(-1,1,0)=-w_{2} .
\end{aligned}
$$

Evidentemente se trata de un paralelogramo en un plano horizontal (la componente $z$ es cero para todos los $w_{i}$ ). 
Finalmente, como un ejemplo de un caso degenerado, usaremos la matriz singular

$$
\mathcal{O}=\left[\begin{array}{lll}
1 & 1 & 0 \\
1 & 1 & 0 \\
0 & 0 & 1
\end{array}\right]
$$

Los vértices $M_{i}$ resultantes son:

$M_{1}=(1,2,0), M_{2}=(1,2,0), M_{3}=(2,1,0)$ y $M_{4}=(2,1,0)$

Los vectores $w_{i}$ son

$$
\begin{aligned}
& w_{1}=(0,0,0), w_{2}=(1,-1,0), \\
& w_{3}=(0,0,0) \text { y } w_{4}=(-1,1,0)
\end{aligned}
$$

En este caso el paralelogramo degeneró en una línea, pues los dos vectores impares son nulos y los dos pares coinciden en sentido contrario.

\section{Regresando a dos Dimensiones}

Para la extensión del Teorema de Varignon en el plano, utilizamos números complejos para representar los segmentos de línea y también para los operadores. Podríamos haber utilizado vectores bidimensionales y para los operadores matrices $2 \times 2$ arbitrarias como lo hicimos para tres dimensiones. Con cualquiera de los dos métodos podemos colocar uno de los vértices del paralelogramo, digamos $M_{1}$, a voluntad. Bastaría, para el caso de números complejos en dos dimensiones, resolver la ecuación

$$
M_{1}=P+q v_{1}
$$

Donde $M_{1}$ lo escogemos a voluntad. Despejando $q$ se obtiene

$$
q=\left(M_{1}-P\right) / v_{1}
$$

El número complejo q se utilizaría para los demás vectores $v_{i}$ y los puntos $Q, R, S$. Los demás vértices del paralelogramo quedarían determinados. Por otra parte, si se utilizan matrices $2 \times 2$ lo que habría que resolver sería la ecuación matricial

$$
M_{l}=P+A v_{1}
$$

donde $M_{1}$ es un vector conocido con 2 componentes (escogido por nosotros), $P$ y $v_{1}$ son vectores dados de dos componentes, y $\mathrm{A}$ es una matriz $2 \times 2$ con cuatro valores desconocidos. Hablando en términos de escalares, tenemos entonces dos ecuaciones lineales simultáneas con cuatro desconocidas, que son las cuatro componentes de la matriz A, por lo que en general habrá una infinidad de soluciones con 2 parámetros arbitrarios. Dichos parámetros se podrían utilizar, por ejemplo, para escoger otro de los puntos del paralelogramo. Este breve análisis parece indicar que utilizar matrices para los operadores nos da mayor flexibilidad que utilizar números complejos. Una evidencia más de que es conveniente tener más de una manera de resolver un problema.

\section{Regresando a tres dimensiones}

Así como se presentaron dos alternativas para generar extensiones al teorema de Varignon en dos dimensiones, en tres dimensiones también existen alternativas adicionales para implantar el operador utilizado. Existe la posibilidad de utilizar la multiplicación por un escalar y el producto cruz o producto vectorial como operador para transformar los vectores $v_{i}$ que se apoyan en los puntos medios de los lados del cuadrilátero para obtener un resultado vectorial. En la expresión $M_{1}=P+\mathcal{O} v_{1}$, así como en dos dimensiones tomamos el producto por un número complejo como un operador, en tres dimensiones se puede escoger la suma de un escalar y un vector tridimensional y usarlos como factor escalar y factor vecto. rial (en un producto cruz) cuyo resultado al actuar sobre un vector es un vector tridimensional. Dado que el producto cruz de dos vectores $v_{a}$ y $v_{b}$ producen un vector perpendicular al plano de ambos, con sentido de acuerdo con la regla del tornillo derecho, y con magnitud vectorial $\left|v_{a}\right|\left|v_{b}\right|\left|\operatorname{sen}\left(v_{a}, v_{b}\right)\right|$, aunque no es conmutativo, si es distributivo con respecto a la suma de vectores (Cannon, 1967), por lo que sin más análisis podemos concluir que los vértices obtenidos con las expresiones

$$
\begin{aligned}
M_{1}= & P+(a+h \times) v_{1}, M_{2}=Q+(a-h \times) v_{2}, M_{3}= \\
& R+(a+h \times) v_{3}, M_{4}=S+(a-h \times) v_{4}
\end{aligned}
$$

donde a es un escalar, los términos entre paréntesis idican una manera simbólica de representar la suma de operadores, por ejemplo, $(\mathrm{a}+\mathrm{h} \times) v_{1}$ significa $a v_{1}+\mathrm{h} \times v_{1}$ que es la suma de dos vectores. Todos los demás símbolos representan vectores tridimensionales y el símbolo " $x$ " representa el producto cruz de vectores tridimensionales, produce vectores $M_{i}$ que representan en el espacio tridimensional los vértices de un paralelogramo.

Cabría hacer un análisis sobre cuál método es más conveniente, si las matrices $3 \times 3$ o la suma de escalares con vectores con tres componentes y el producto cruz. Aunque no lo detallemos, resulta que con los dos métodos podemos escoger uno de los vértices del paralelogramo, digamos $M_{1}$, 
pero la matriz $3 \times 3$ nos da una flexibilidad adicional, ya que la determinación de la matriz necesaria para fijar $M_{1}$ nos deja varios parámetros libres para otros posibles propósitos*.

\section{Conclusiones}

Iniciamos este artículo con dos ideas:

1) Que vale la pena tener más de un método para resolver un problema. Esto lo ilustramos con tres métodos: el método geométrico elemental, los números complejos para la geometría plana y los vectores tridimensionales para geometría sólida. El pasar de un método a otro nos ayudó a visualizar posibles extensiones y generalizaciones de los teoremas de Pitágoras y de Varignon.

2) Que cerca de una buena idea generalmente hay otras. En otras palabras, las buenas ideas llegan en racimos. Dos ideas que ilustran esta aseveración son los teoremas de Pitágoras y el Teorema de Varignon. Sus extensiones y generalizaciones son parte del mismo racimo. El teorema de Pitágoras y el concepto ángulo recto (o vectores ortogonales) se ha extendido (no en este artículo) a cuatro, cinco y más dimensiones y también a un número infinito, tanto contable como continuo de dimensiones. Lo mismo con el concepto de ángulo entre dos vectores, los conceptos de producto interno o producto punto, longitud de un vector y distancia entre dos puntos, han dado origen a espacios euclideanos $n$ - dimensionales y espacios de Hilbert tanto discretos $\left(l^{2}\right)$ como continuos ( $\left.L^{2}\right)$ que tienen muy importantes aplicaciones científicas y tecnológicas (Taylor, 1958). El método de trabajar con vectores y números complejos en geometría plana nos permite demostrar ciertos teoremas con más generalidad sin tener que hacer demostraciones para diversos casos. Nos inspira, por otra parte, a definir áreas negativas dependiendo de la manera como se recorren los vectores que encierran el área (Klein, 1939). Por ejemplo, el cuadrilátero con vértices $\mathrm{A}, \mathrm{B}, \mathrm{C}$ y $\mathrm{D}$ que se muestra en la figura 21 cuyos lados se cruzan sin formar vértices, tiene área menor que la suma de las áreas convencionales de los dos triángulos que se forman, porque la porción mayor se encierra recorriendo su frontera en dirección contra horaria (positiva) y la otra porción menor se encierra

\footnotetext{
* El producto cruz $a \times b$ entre dos vectores $a=\left(a_{1}, a_{2}, a_{3}\right)$ y $b=\left(b_{1}, b_{2}, b_{3}\right)$ es igual al determinante de tercer orden con filas $i, j, k ; a_{1}, a_{2}, a_{3} ; b_{1}, b_{2}, b_{3}$ donde $i, j, k$ son vectores unitarios (Thomas, 1960). Si suponemos dado el segundo vector y buscamos el primero para obtener un resultado deseado (para fijar, digamos, $M_{1}$ ), es necesario satisfacer tres ecuaciones lineales simultáneas cuyo determinante es idénticamente nulo, lo cual, le pone restricciones a los dos vectores predeteminados para que exista solución. Al incluir el escalar $a$, la matriz deja de ser singular y se puede escoger $M_{1}$ a voluntad. En todo el artículo se ignora la posibilidad de sumar a todos los vértices un vector arbitrario independiente del cuadrilátero original, pues eso lo único que haría sería trasladar todo el paralelogramo paralelo a sí mismo, por lo tanto en el asunto de fijar $M_{1}$, se ignora esta posibilidad.
}

recorriendo su frontera en sentido horario (negativo). El teorema de geometría elemental que dice: "El área de un trapezoide es igual a su altura (distancia entre los dos lados paralelos del trapezoide) por la longitud de su bimediana (línea que une los centros de los dos lados opuestos no paralelos)" (Jurgensen et al., 1963) sólo es válido para trapezoides convexos, por lo que no se aplica a la figura que aparece en la figura 21. Sin embargo, si definimos áreas negativas en caso de que se encierren con un recorrido horario y positivas cuando es contra horario, entonces sí es válido calcular el área neta (suma algebraica de las áreas) multiplicando la altura por la longitud de la bimediana.

En el artículo no se ha agotado el tema de extender y generalizar el Teorema de Varignon. Una extensión adicional se le puede aplicar a los triángulos. En la figura 22 se muestra un triángulo que se puede considerar como un caso particular de un cuadrilátero con uno de los lados con longitud cero. El lado que se anula se reduce a un punto y su centro es el propio punto. La aplicación del Teorema de Varignon nos produce el paralelogramo que se muestra. Las extensiones y generalizaciones del Teorema de Varignon que se dan en este artículo se aplican por lo tanto también a triángulos como casos particulares de cuadriláteros con un lado reducido a un punto. La justificación se basa en que la demostración se hizo simbólicamente y no se requirió poner restricciones a los vectores $v_{i}$, éstos se pueden anular sin problema y las figuras no necesitan ser convexas.

Otros teoremas con cuadriláteros más restringidos se pueden establecer con facilidad usando las técnicas de uso de números complejos utilizadas en este artículo,

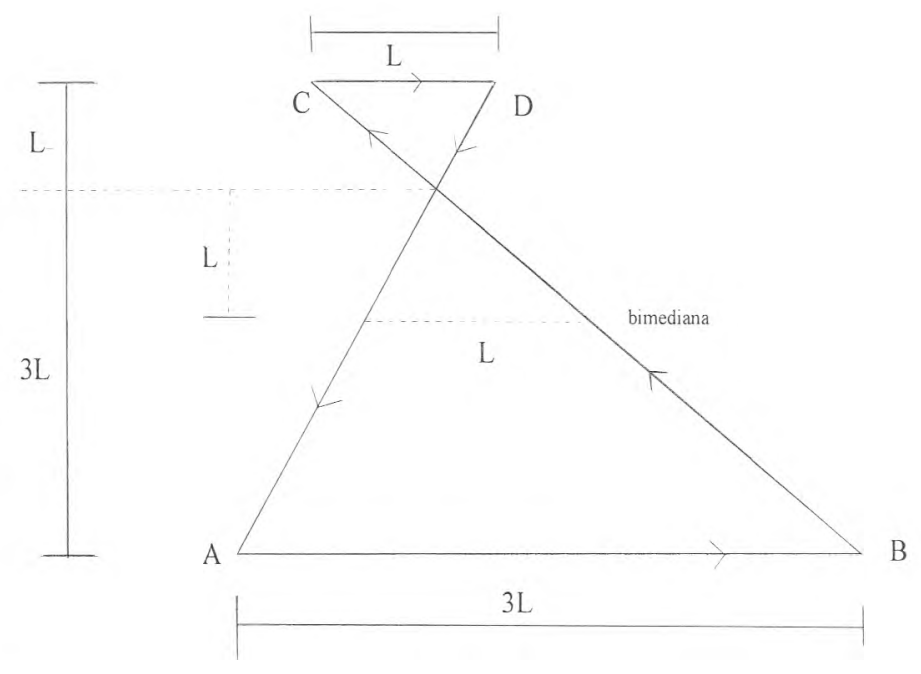

Figura 21

Área Neta $=3 \mathrm{~L} \times 3 \mathrm{~L} / 2-\mathrm{L} \times \mathrm{L} / 2=4 \mathrm{~L}^{2}$

Área con Teorema de la altura y la bimediana $=\mathrm{L} \times 4 \mathrm{~L}=4 \mathrm{~L}^{2}$ 


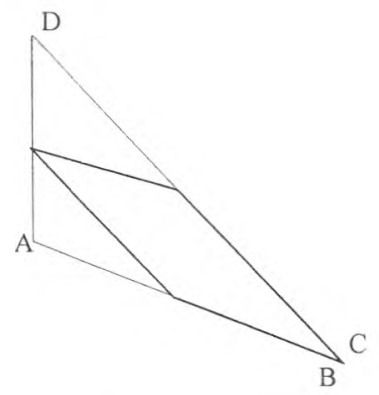

Figura 22

así, en la figura 23 se muestra un paralelogramo arbitrario al cual, si se le construyen hacia fuera (o hacia dentro) cuadrados a los lados y se unen los centros de los cuadrados, la figura resultante es un cuadrado. (Yaglom, 1962) y (Murray-Lasso, 2000) muestran el Teorema de Von Aubel, que aunque no es extensión del Teorema de Varignon se demuestra con técnicas muy similares).

Esqueleto de Demostración Al representar los lados del (c) paralelogramo con números complejos $2 z_{1}, 2 z_{2}, 2 z_{3}, 2 z_{4}, y$ las líneas que van de los centros de los cuadrados sobre los lados con $-i z_{1}$ (o con $i z_{i}$ si van hacia adentro) los lados $w_{i}$ del cuadrado grueso quedan representados por números complejos que van entre centros de cuadrados de lados contiguos. Resulta que $w_{2}=i w_{1}$, $w_{4}=i w_{3}, w_{3}=-w_{1}, w_{4}=-w_{2}$, lo cual demuestra que la figura con vértices en los centros de los cuadrados es un cuadrado.

\section{Figura 23}

Otra dirección en la que no agotamos posibilidades de extensiones y generalizaciones del Teorema de Varignon son los espacios n-dimensionales. Habiendo establecido que el teorema es válido para dos y tres dimensiones, esencialmente con la misma demostración, es muy fácil irse a espacios de más dimensiones y utilizando los mismos símbolos con diferente interpretación repetir las demostraciones introduciendo operadores representados por matrices $\mathrm{n} \times \mathrm{n}$ que representan transformaciones lineales generales en los espacios de $n$ dimensiones. Los paralelogramos serán figuras de cuatro lados con pares de lados opuestos paralelos y de igual longitud en los espacios n-dimensionales. Incidentalmente, el concepto de longitud en estos espacios utiliza el Teorema de Pitágoras extendido a dichos espacios.
El Teorema de Pitágoras también admite otras extensiones y generalizaciones además de las ya mencionadas en el artículo. Una dirección que merece ser investigada como extensión del Teorema de Pitágoras es el "tetraedro trirrectángulo" con vértices A, B, C, D como se muestra en la figura 24. En dicho tetraedro se conocen las áreas A, B y C de las tres caras sobre los planos coordenados opuestas a los vértices $A, B, C$ que son incidentes en el vértice trirrectangular $\mathrm{D}$. Se desea conocer el área $\mathrm{D}$ de la cara oblicua opuesta al vértice $D$. (Nótese la analogía de las caras de áreas $\mathrm{A}, \mathrm{B}$ y C con los catetos de un triángulo rectángulo y de la cara de área D con la hipotenusa). Polya (1981), demuestra con técnicas de geometría elemental que

$$
D^{2}=A^{2}+B^{2}+C^{2}
$$

y le llama a esta relación "el análogo del Teorema de Pitágoras en geometría sólida.”

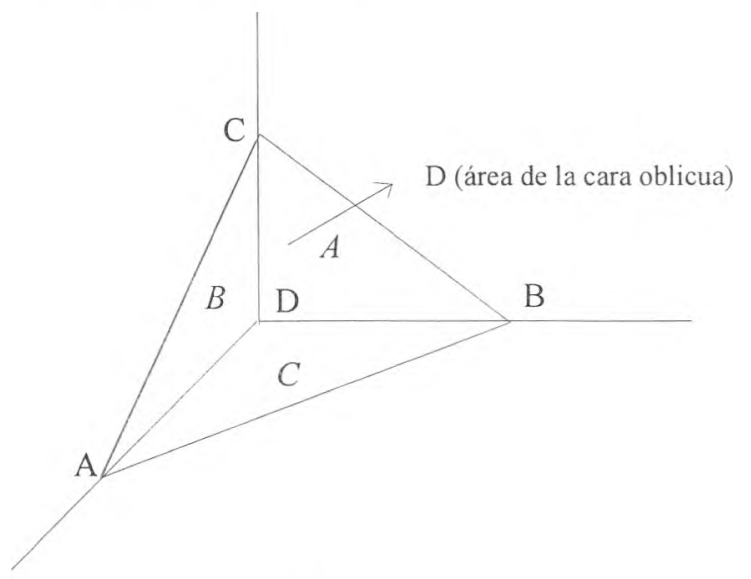

Figura 24

Un punto a investigar es la posible representación de áreas planas por medio de vectores normales a sus planos y la interpretación de los "polígonos suma" de dichos vectores. Algo que se ve promisorio y generalizable a n dimensiones es la teoría que tiene que ver con proyecciones ortogonales de superficies e hipersuperficies.

La geometría siempre ha sido importante en ingeniería; sin embargo, con el avance de la tecnología, especialmente la tecnología informática, ha aumentado notablemente su importancia en las aplicaciones. Entre las áreas en las cuales esto ha sucedido tenemos la graficación por computadora, el modelado de superficies tridimensionales, la geometría computacional (por ejemplo, decidir en qué celda está un punto, o en qué municipio de la república Mexicana está un punto de coordenadas geográficas dadas), las aplicaciones de la geometría diferencial en el modelado de medios continuos en áreas como la elasticidad y plasticidad, mecánica de suelos, hidrodinámica, 
meteorología, electromagnetismo, estudios de estratificación del suelo en la búsqueda de petroleo, agua y minerales, estudios de terreno en la explotación y exploración petrolera, la robótica y en general la cinemática y el diseño de mecanismos, el CAD/CAM, el dibujo mecánico, la elaboración de planos por computadora, el diseño arquitectónico, el diseño gráfico publicitario, el diseño urbano, los sistemas de información geográfica, las artes gráficas, la tipografía, el procesamiento digital de imágenes, la navegación aérea y marítima, el ajuste de curvas, la cartografía, la topografía, la elaboración de mapas por cualquier método, la animación por computadora, el cine por computadora, la tomografía y otros métodos de imagenología médica, la visualización computarizada, la fotogrametría, la estimación de volúmenes y áreas en agrimensura y construcción, los sistemas de posicionamiento global por satélite, problemas de posicionamiento óptimo, el diseño de plantas industriales, los diagramas de Voronoi, la triangulación de Delaunay.

No obstante lo anterior, en las carreras de ingeniería no existe ningún curso de geometría (excepto geometría analítica y en algunas carreras geometría descriptiva); en general el último curso de geometría (excluyendo las mencionadas) que toma un alumno de ingeniería corresponde a secundaria. Es necesario a la luz de las aplicaciones recientes, remediar esta situación en los planes de estudio introduciendo cursos modernos que combinen la geometría con la computación y las aplicaciones (Meyer, 1999).

\section{Referencias}

Cannon R.H. Jr. (1967). Dynamics of Physical Systems. McGraw-Hill Book Company, New York.

Coxeter H.S.M. (1971). Fundamentos de geometría. Limusa-Wiley, México.

Davis P.J. y Hersh R. (1981). The Mathematical Experience. Houghton Mifflin Company, Boston, MA.
Heath T.L. (1956). The Thirteen Books of Euclid's Elements (3 Vols.). Dover Publications, Inc., New York.

Jurgensen R.C., Donnelly A.J. y Dolciani M.P. (1963). Modern Geometry: Structure and Method. Houghton Mifflin Company, Boston, MA.

Klein F. (1939). Elementary Mathematics from an Advanced Viewpoint: Geometry. Dover Publications, New York.

Logo Computer Systems, Inc. (1990). LogoWriter: Guía de referencia. Macrobit Editores, México.

Lyúbich Yu.I y Shor L.A. (1978). Método cinemático en problemas geométricos. Editorial Mir, Moscú.

Meyer W. (1999). Geometry and Its Applications. Harcourt Academic Press, San Diego, CA.

Modenov P.S. y Parkhomenko A.S. (1965). Geometric Transformations, Vol. I: Euclidean and Affine Transfor. mation. Academic Press, New York.

Moise E.E. y Downs F.L. Jr. (1975). Geometry. Addison-Wesley Publishing Company, Menlo Park, CA.

Moise E.E. (1968). Elementos de geometría superior. Compañía Editorial Continental, SA, México.

Murray-Lasso M.A. (2000). La aplicación de los números complejos en la enseñanza de la geometría plana. Ingeniería: Investigación y Tecnología, Vol. 1, No. 5, octubre-diciembre, 215-223.

Papert S. (1980). Mindstorms: Children, Computers, and Powerful Ideas. Basic Books, New York. Versión en español: Papert S. (1981). Desafío a la mente: Computadoras y educación, Ediciones Galápago, Buenos Aires.

Papert S. (1993). The Children's Machine: Rethinking School in the Age of the Computer.Basic Books, New York.

Polya G. (1981). Mathematical Discovery: On Understanding, Learning and Teaching Problem Solving, Vols. I, II, Combined Edition. John Wiley \& Sons, Inc., New York.

Taylor A.E. (1958). Introduction to Functional Analysis. John Wiley \& Sons, Inc. New York.

Thomas G.B. Jr. (1960). Calculus and Analytic Geometry (Third Edition), Addison-Wesley Publishing Company, Inc., Reading, MA.

Yaglom I.M. (1962). Geometric Transformations. New Mathematical Library, Random House, New York.

\section{Semblanza del autor}

Marco Antonio Murray-Lasso. Realizó la licenciatura en ingeniería mecánica-eléctrica en la Facultad de Ingeniería de la UNAM. El Instituto de Tecnología de Massachussetts (MIT) le otorgó los grados de maestro en ciencias en ingeniería eléctrica y doctor en ciencias cibernéticas. En México, ha laborado como investigador en el Instituto de Ingeniería y como profesor en la Facultad de Ingeniería (UNAM) durante 39 años; en el extranjero, ha sido asesor de la NASA en diseño de circuitos por computadora para aplicaciones espaciales, investigador en los Laboratorios Bell, así como profesor de la Universidad Case Western Reserve y Newark College of Engineering, en los Estados Unidos. Fue el presidente fundador de la Academia Nacional de Ingeniería de México; vicepresidente y presidente del Consejo de Academias de Ingeniería y Ciencias Tecnológicas (organización mundial con sede en Washington que agrupa las Academias Nacionales de Ingeniería) y secretario de la Academia Mexicana de Ciencias. Actualmente, es jefe de la Unidad de Enseñanża Auxiliada por Computadora de la División de Estudios de Posgrado de la Facultad de Ingeniería de la UNAM, investigador nacional en ingeniería, consejero educativo del MIT y consultor de la UNESCO. 
DOI: http://dx.doi.org/10.22201/fi.25940732e.2001.02n4.017 